\title{
Approximating GARCH-Jump Models, Jump-Diffusion Processes, and Option Pricing *
}

\author{
Jin-Chuan. Duan $^{\dagger} \quad$ Peter Ritchken ${ }^{\ddagger}$ Zhiqiang Sun ${ }^{\S}$
}

March 19, 2005

(to appear in Mathematical Finance)

\footnotetext{
${ }^{*}$ This paper benefits from the comments by the two anonymous reviewers and the participants of the 2004 American Finance Association meetings in San Diego, the Daiwa International Workshop in Financial Engineering held in Tokyo and Kyoto in August 2004, the 2004 Annual Derivatives Conference in New York, the Conference on Financial and Economic Policies and Financial Engineering in Taipei in May 2004, the Workshop on Mathematical Finance and Insurance in Huangshan, China in May 2004 and the Risk Management in Insurance Conference at University of Waterloo in June 2004. The authors also thank the seminar participants at Providence University and the National Center for Theoretical Sciences in Taiwan for their comments. Financial support from both the Social Sciences and Humanities Research Council of Canada and the Natural Sciences and Engineering Research Council of Canada is gratefully acknowledged.

${ }^{\dagger}$ Rotman School of Management, University of Toronto, Ontario, Canada, M5S 3E6. Email jcduan@rotman.utoronto.ca.

${ }^{\ddagger}$ Weatherhead School of Management, Case Western Reserve University, Cleveland, Ohio, 44106. Email phr@po.cwru.edu.

${ }^{\S}$ Weatherhead School of Management, Case Western Reserve University, Cleveland, Ohio, 44106. Email zxs9@po.cwru.edu
} 


\title{
Approximating GARCH-Jump Models, Jump-Diffusion Processes, and Option Pricing
}

\begin{abstract}
This paper considers the pricing of options when there are jumps in the pricing kernel and correlated jumps in asset prices and volatilities. We extend theory developed by Nelson (1990) and Duan (1997) by considering limiting models for our resulting approximating GARCH-Jump process. Limiting cases of our processes consist of models where both asset price and local volatility follow jump diffusion processes with correlated jump sizes. Convergence of a few GARCH models to their continuous time limits are evaluated and the benefits of the models explored.

(GARCH option models, stochastic volatility models with jumps, limiting GARCH with Jump processes)
\end{abstract}


Up until the 1990s the literature on continuous time models, used in theoretical finance and especially in derivative modeling, and discrete time models, often favored in empirical studies, developed along very separate lines. Most of the discrete time models were of the generalized autoregressive conditionally heteroskedastic $(\mathrm{GARCH})$ type, while the continuous time models were based on diffusion models. In the early 1990s researchers began to reconcile the two approaches. In particular, Nelson (1990) showed that as the sampling frequency increased, the volatility process generated within some GARCH models converged in distribution towards well defined solutions of stochastic differential equations. Duan (1997) extended this work and showed that most of the existing bivariate diffusion models that had been used to model asset returns and volatility could be represented as limits of a family of GARCH models. As a result, even if one prefers modeling prices and volatilities by a bivariate process, there may be advantages in considering GARCH techniques. For example, by suitably curtailing the parameters of generalized GARCH processes, we can obtain European and American option prices under the stochastic volatility models of Hull and White (1987), Scott (1987), Wiggins (1987), Stein and Stein (1991), and Heston (1993).

Unfortunately, there is now overwhelming empirical evidence that indicates that stock price processes cannot be adequately characterized by bivariate diffusions, and that incorporating jump components in both price and in volatility is necessary. For example, Bates (2000) and Pan (2002) consider models where prices follow a jump-diffusion process with volatility being characterized by a correlated diffusive stochastic process. Both authors show that such models are incapable of capturing empirical features of equity index returns or option prices, and they attribute this to the fact that volatility itself may contain jumps. More recently, Eraker, Johannes and Polson (2003) examine the jump in volatility models proposed by Duffie, Singleton and Pan (1999), and provide a study that shows that the addition of jumps in volatility provide a significant improvement to explaining the returns data on the S\&P 500 and Nasdaq 100 index, beyond a stochastic volatility model with just jumps in prices.

To date, the GARCH approximating models that have been considered in the literature are set up for stochastic volatility diffusions. In light of the importance of jumps, both in price and volatility, the current GARCH approximating models are inadequate. The primary purpose of this paper is to propose a new set of GARCH models that include, as limiting cases, processes characterized by stochastic volatility with jumps in price and volatility. The secondary purpose of this paper is to explore the potential advantages of these GARCH with jump models over their continuous time counterparts.

The discrete time model on which we rely in constructing our limiting models is that of Duan, Ritchken and Sun (2004) (hereafter DRS). The DRS (2004) model has the property that the conditional returns have fat tails and are skewed. As a result, local skewness and kurtosis present in data can easily be matched. Further, much of the volatility smile observed in option 
prices can be well explained by the DRS (2004) model. In addition, estimating the parameters of the DRS (2004) can be readily accomplished using historical time series data and/or panels of option prices, using standard maximum likelihood estimation techniques. This simplicity stands in contrast to the rather delicate task of estimating parameters for continuous-time processes when the volatility is a latent, nonobservable state variable, which, together with price, contain diffusive and jump elements. ${ }^{1}$

We develop an approximating GARCH-Jump option pricing model that can be viewed in parallel with the discrete time binomial option pricing models. Specifically, the binomial model, which has the ability to approximate a variety of different diffusive or jump processes, depending on how the limits are taken, serves as a work-horse for pricing European and American options. We will show that our approximating GARCH-Jump model has the ability to lead to a wide variety of stochastic volatility models that incorporate jumps and diffusive elements in prices and volatilities, and also has the property that option prices can be relatively efficiently computed.

While our approximating GARCH-Jump models can be viewed as filters for continuoustime stochastic volatility models with jumps in price and volatility, they also may be useful for approximating the continuous time stochastic volatility models with jumps. Indeed, we find that option prices generated under certain GARCH-Jump processes may converge to the theoretical continuous time option prices faster than option prices computed under Euler discretization schemes of the continuous time processes. As a result, even if one believes the true process to be a continuous-time jump diffusion process for both price and volatility, our approximating GARCH-Jump models may still be useful because they could provide excellent numerical schemes for computing option prices under the true processes.

One reason for considering limiting models is to take advantage of the convenient analytical properties of the limiting model; for example, the limiting model may enable the derivation of a closed-form pricing formula for certain options. When the discrete-time model becomes increasingly complex to accommodate empirical data features, its corresponding limiting model is expected to lose its analytical tractability. Indeed, the three limiting models presented in this paper do not appear to have closed-form pricing solutions even for European options. Lack of a closed-form solution need not be considered a weakness. There is no closed-form pricing formula for the American-style options in the constant-volatility Black-Scholes framework. However, it does not impede the Black-Scholes model's applicability because reliable numerical methods

\footnotetext{
${ }^{1}$ While in the last decade, significant advances in econometric methodology have been made, these estimation problems are still fairly delicate. Eraker, Johannes and Polson (2003) provide an excellent review of the difficulties in adopting standard MLE or GMM approaches. Singleton (2001) discusses an approach using characteristic functions. An alternative approach based on simulation methods using Efficient Method of Moments, and Monte Carlo Markov Chains does resolve some of these issues. For an overview on econometric techniques to estimate continuous-time models see Renault (1997), Jacquier, Polson, and Rossi (1994), Eraker, Johannes and Polson (2003), and the references therein.
} 
are available for such options. In short, the purpose of obtaining limiting models in this paper has little to do with closed-form solutions. ${ }^{2}$ Rather, we want to gain further theoretical understanding on a new class of continuous-time option models involving jumps in volatility from a discrete-time perspective, or vice versa. Furthermore, we want to rely on such a theoretical linkage to open up the possibility of employing the convenient maximum likelihood estimation method and the numerical pricing techniques associated with the GARCH family of models for a new class of continuous time models with jumps in volatility. ${ }^{3}$

The paper proceeds as follows. In the first section we establish the basic setup for the pricing kernel and for the dynamics of the asset price over ever-shrinking trading intervals. This approximating model extends the DRS (2004) model by introducing the element of interval length. Our generalization of the DRS (2004) model allows for exploring limiting behavior. In section 2 we examine in detail some limiting cases of our model. We focus on models that converge to processes containing diffusive and jump elements in both prices and/or volatilities. When jumps are shut down in our model, then we are left with standard approximating GARCH processes for which it is possible to obtain limiting forms that lead to options being priced as in Heston (1993), Hull and White (1987), Scott (1987), and others. Further, when our approximating GARCH process is curtailed so that volatility is no longer stochastic, but jumps allowed in prices, our limiting model nests the jump-diffusion model of Merton (1976), or the more general model of Naik and Lee (1990). Of interest in this paper, however, is the more general case, when our limiting models have both diffusive and jump elements in prices and volatilities. This allows us to have proxies for the time series models used in Eraker, Johannes and Polson (2003) as well as for jump-diffusion options priced along the lines of Duffie, Singleton and Pan (1999). For the most part we focus on equilibrium models where the dynamics of prices under both the physical process as well as the risk neutral process are well specified. This allows us to use standard maximum likelihood estimation on the time series, as well as panel data of options, to readily estimate and/or calibrate the parameters. However, as is fairly common in the derivatives literature, we could focus on the risk neutral process alone. If we do this, then less structure is required in the drift term of the asset, and our approximating GARCH with jumps models can be further generalized. In section 3 we conduct simulation experiments designed to provide an indication of the speed of convergence of option prices. We compare prices obtained by Euler and our GARCH-Jump approximating schemes, with true prices, and demonstrate the speed of convergence of the GARCH-Jump prices to their jump-diffusion limits.

\footnotetext{
${ }^{2}$ The notion of closed-form solutions has in the literature been subjected to a wide range of interpretations. For example, the "closed-form" stochastic-volatility option pricing model of Heston (1993) requires numerically evaluating a Fourier inversion, and the Fourier inversion method does not work for American and/or exotic options. The "closed-form" GARCH option model of Nandi and Heston (2001) requires numerically solving a difference equation system in addition to a numerical Fourier inversion, and is also limited to European options.

${ }^{3}$ The GARCH-based numerical pricing techniques are, for example, Ritchken and Trevor (1999) and Duan and Simonato (2001).
} 
Section 4 concludes.

\section{The Basic Setup}

We consider a discrete-time economy for a period of $[0, T]$ where trading takes place at any of the $n+1$ trading points $0, \Delta t, 2 \Delta t \ldots, n \Delta t$ where $\Delta t=\frac{T}{n}$. Uncertainty is defined on a complete filtered probability space $(\Omega, \mathcal{F}, \mathrm{P})$ with filtration $\mathbf{F}=\left(\mathcal{F}_{t}\right)_{t \in\{0, \Delta t, 2 \Delta t, \ldots, n \Delta t=T\}}$ where $\mathcal{F}_{0}$ contains all P-null sets in $\mathcal{F}$. Let $m_{i \Delta t}$ be the marginal utility of consumption at date $i \Delta t$.

For pricing to proceed, the joint dynamics of the asset price, and the pricing kernel, $\frac{m_{i \Delta t}}{m_{(i-1) \Delta t}}$, needs to be specified. We have

$$
S_{i \Delta t}=E^{\mathrm{P}}\left[S_{i \Delta t} \frac{m_{i \Delta t}}{m_{(i-1) \Delta t}} \mid \mathcal{F}_{(i-1) \Delta t}\right]
$$

where $S_{i \Delta t}$ is the total payout, consisting of price and dividends. The expectation is taken conditional on the information up to date $(i-1) \Delta t$, under the physical measure P.

We assume that the dynamics of this pricing kernel, $m_{i \Delta t} / m_{(i-1) \Delta t}$, is given by:

$$
\frac{m_{i \Delta t}}{m_{(i-1) \Delta t}}=e^{a(\Delta t)+b J_{i}(\Delta t) \sqrt{\Delta t}}
$$

where $J_{i}(\Delta t)$ is a standard normal random variable plus a Poisson random sum of normally distributed variables. That is,

$$
J_{i}(\Delta t)=X_{i}^{(0)}+\sum_{j=1}^{N_{i}(\Delta t)} X_{t}^{(j)}(\Delta t)
$$

where $X_{i}^{(0)} \sim N(0,1)$ and $X_{i}^{(j)}(\Delta t) \sim N\left(\mu(\Delta t), \gamma^{2}(\Delta t)\right)$ for $j=1,2, \ldots$, and $N_{i}(\Delta t), i=1,2, \ldots n$ are a sequence of independent Poisson random variables with parameter $\lambda \Delta t$. Although we have assumed a constant $\lambda$, our theoretical results remain valid if the Poisson parameter is stochastic, and at each date, $i \Delta t$, is $\mathcal{F}_{(i-1) \Delta t}$-measurable. The random variables, $X_{i}^{(j)}$, are independent for $j=0,1,2 \ldots$ and $i=1,2, \ldots, n$.

The asset price, $S_{i \Delta t}$, is assumed to follow the process:

$$
\frac{S_{i \Delta t}}{S_{(i-1) \Delta t}}=e^{f_{i \Delta t}(\Delta t)+\sqrt{h_{i \Delta t}} \bar{J}_{i}(\Delta t) \sqrt{\Delta t}}
$$

where $\bar{J}_{i}(\Delta t)$ is a standard normal random variable plus a Poisson random sum of normal random variables. In particular:

$$
\bar{J}_{i}(\Delta t)=\bar{X}_{i}^{(0)}+\sum_{j=1}^{N_{i}(\Delta t)} \bar{X}_{i}^{(j)}(\Delta t)
$$


where $\bar{X}_{i}^{(0)} \sim N(0,1)$ and $\bar{X}_{i}^{(j)}(\Delta) \sim N\left(\bar{\mu}(\Delta t), \bar{\gamma}^{2}(\Delta t)\right)$ for $j=1,2, \ldots$ The random variables, $\bar{X}_{i}^{(j)}$, are independent for $j=0,1,2 \ldots$ and $i=1,2, \ldots, n$. Furthermore, for $i=1,2, \ldots, n$ :

$$
\operatorname{Corr}\left(X_{i}^{(j)}, \bar{X}_{i^{\prime}}^{\left(j^{\prime}\right)}\right)= \begin{cases}\rho & \text { if } i=i^{\prime} \text { and } j=j^{\prime} \\ 0 & \text { otherwise }\end{cases}
$$

and $N_{i}(\Delta t), i=1,2 . ., n$ is the same sequence of Poisson random variables as in the pricing kernel.

The expected value, $E^{\mathrm{P}}\left(\bar{J}_{i}(\Delta t) \mid \mathcal{F}_{(i-1) \Delta t}\right)$, and variance, $\operatorname{Var}^{\mathrm{P}}\left(\bar{J}_{i}(\Delta t) \mid \mathcal{F}_{(i-1) \Delta t}\right)$, of $\bar{J}_{i}(\Delta t)$ are

$$
\begin{aligned}
E^{\mathrm{P}}\left(\bar{J}_{i}(\Delta t)\right) & =\lambda \bar{\mu}(\Delta t) \Delta t \\
\operatorname{Var}^{\mathrm{P}}\left(\bar{J}_{i}(\Delta t)\right) & =1+\lambda \hat{\gamma}^{2}(\Delta t) \Delta t
\end{aligned}
$$

where $\hat{\gamma}^{2}(\Delta t)=\bar{\mu}^{2}(\Delta t)+\bar{\gamma}^{2}(\Delta t)$.

The Poisson random variable provides a random number of shocks in period $i$. Given that the number of shocks in a particular period is some nonnegative integer $k$, say, the logarithm of the pricing kernel for that period consists of a draw from the sum of $k+1$ normal distributions, while the logarithmic return of the asset also consists of a draw from the sum of $k+1$ normal random variables that are correlated with the elements in the pricing kernel. In either case, the first normal random variable is standardized to have mean 0 and variance 1 because its location and scale have already been reflected in the model specification. The structure for the means and variances, $\bar{\mu}(\cdot)$ and $\bar{\gamma}^{2}(\cdot)$ as functions of time are the same as those for $\mu(\cdot)$ and $\gamma(\cdot)$ in the pricing kernel.

The local variance of the logarithmic returns for date $i \Delta t$, viewed from date $(i-1) \Delta t$ is $h_{i \Delta t} \operatorname{Var}\left(\bar{J}_{i \Delta t}\right) \Delta t=h_{i \Delta t}\left(1+\lambda \hat{\gamma}^{2}(\Delta t) \Delta t\right) \Delta t$. We shall refer to $h_{i \Delta t}$ as the local scaling factor because it differs from local variance by a constant. In general, the local scaling factor, $h_{i \Delta t}$, can be any predictable process. We shall assume,

$$
h_{(i+1) \Delta t}=H\left(h_{i \Delta t}, \bar{J}_{i \Delta t}\right)
$$

DRS (2004) considered a special case of this model with $\Delta t=1$, and an updating process of the non-linear asymmetric GARCH (NGARCH) form:

$$
h_{i+1}=\beta_{0}+\beta_{1} h_{i}+\beta_{2} h_{i}\left(\frac{\bar{J}_{i}-E^{\mathrm{P}}\left(\bar{J}_{i}\right)}{\sqrt{\operatorname{Var} \mathrm{P}\left(\bar{J}_{i}\right)}}-c\right)^{2} .
$$

Here $\beta_{0}$ is positive, and $\beta_{1}$ and $\beta_{2}$ are nonnegative to ensure that the local scaling process is positive. $\bar{J}_{i}$ in the last term is normalized to make this equation comparable to the NGARCH model which typically uses a random variable with mean 0 and variance 1 . Notice that when 
$\lambda=0$ the model reduces to the NGARCH-Normal process used by Duan (1995). When $\lambda$ is released from 0 , the innovations are a random mixture of normals. This model, called the GARCH-Jump model, was empirically tested by DRS (2004), who showed that the inclusion of "jumps" significantly improved the fit of historical time series of the S\&P 500, as well as helping to explain a significant portion of the volatility smile in option prices. Note that by Duan (1997), the process in equation (10) is strictly stationary if $\beta_{1}+\beta_{2}\left(1+c^{2}\right) \leq 1$. The unconditional mean of $h_{i}$ is finite and equals $\beta_{0} /\left[1-\beta_{1}-\beta_{2}\left(1+c^{2}\right)\right]$ if $\beta_{1}+\beta_{2}\left(1+c^{2}\right)<1$. The model we consider is more general, in that the dependence on time increments is made explicit. For example, one of the simplest updating schemes we consider has the form:

$$
h_{(i+1) \Delta t}-h_{i \Delta t}=\beta_{0} \Delta t+h_{i \Delta t}\left(\beta_{1}-1\right) \Delta t+\beta_{2} h_{i \Delta t}\left(\frac{\bar{J}_{i}(\Delta t)-E^{\mathrm{P}}\left(\bar{J}_{i}(\Delta t)\right)}{\sqrt{\operatorname{Var}^{\mathrm{P}}\left(\bar{J}_{i}(\Delta t)\right)}}-c\right)^{2} \Delta t .
$$

Notice that when $\Delta t=1$, the updating equation reduces to the standard NGARCH-Jump model given by equation (10).

For simplicity, we assume that the continuously compounded risk-free interest rate is constant, say, $r{ }^{4}$ Given the dynamics of the pricing kernel and the stock price, the following restrictions must hold:

$$
\begin{aligned}
E^{\mathrm{P}}\left(\frac{m_{i \Delta t}}{m_{(i-1) \Delta t}} \mid \mathcal{F}_{(i-1) \Delta t}\right) & =e^{-r \Delta t} \\
E^{\mathrm{P}}\left(\frac{m_{i \Delta t}}{m_{(i-1) \Delta t}} \frac{S_{i \Delta t}}{S_{(i-1) \Delta t}} \mid \mathcal{F}_{(i-1) \Delta t}\right) & =1 .
\end{aligned}
$$

These equilibrium conditions impose a specific form on the function, $a(\Delta t)$ and $f_{i \Delta t}(\Delta t)$. In particular, substituting equation (2) into (12) uniquely identifies $a(\Delta t)$. Further substituting equations (2) and (4) into (13), leads eventually to the following restriction on $f(\cdot)$ :

$$
f_{i \Delta t}(\Delta t)=\left(r-\frac{h_{i \Delta t}}{2}-b \rho \sqrt{h_{i \Delta t}}\right) \Delta t+\lambda \kappa(\Delta t)\left(1-K_{i}\left(h_{i \Delta t}\right)\right) \Delta t
$$

where

$$
\begin{aligned}
\kappa(\Delta t) & =e^{b \mu(\Delta t) \sqrt{\Delta t}+\frac{b^{2} \gamma^{2}(\Delta t)}{2} \Delta t} \\
K_{i}\left(h_{i \Delta t}\right) & =e^{\sqrt{h_{i \Delta t}}(\bar{\mu}(\Delta t) \sqrt{\Delta t}+b \rho \gamma(\Delta t) \bar{\gamma}(\Delta t) \Delta t)+\frac{h_{i \Delta t}}{2}\left(\bar{\gamma}^{2}(\Delta t) \Delta t\right)} .
\end{aligned}
$$

To summarize, then, under the physical measure, $\mathrm{P}$, we have:

\footnotetext{
${ }^{4}$ Note the constant interest rate assumption is consistent with the pricing kernel specification in equation (2) but not a necessity. We make this assumption so that there is no need to specify an additional stochastic process for the interest rate.
} 
$[\mathrm{P} 1]$

$$
\begin{array}{llc}
S_{i \Delta t} & = & S_{(i-1) \Delta t} e^{f_{i \Delta t}(\Delta t)+\sqrt{h_{i \Delta t}} \bar{J}_{i}(\Delta t) \sqrt{\Delta t}} \\
h_{(i+1) \Delta t} & = & H\left(h_{i} \Delta t, \bar{J}_{i}(\Delta t)\right)
\end{array}
$$

where

$$
\begin{aligned}
\bar{J}_{i}(\Delta t) & =\bar{X}_{i}^{(0)}+\Sigma_{j=1}^{N_{i}(\Delta t)} \bar{X}_{i}^{(j)}(\Delta t) \text { for } i=1,2, \ldots, n \\
\bar{X}_{i}^{(0)} & \sim N(0,1) \\
\bar{X}_{i}^{(j)}(\Delta t) & \sim N\left(\bar{\mu}(\Delta t), \bar{\gamma}^{2}(\Delta t)\right) \text { for } j=1,2, \ldots \\
N_{i}(\Delta t) & \sim \operatorname{Poisson}(\lambda \Delta t)
\end{aligned}
$$

and $f_{i \Delta t}(\Delta t)$ is defined by equation (14). The dynamics of the pricing kernel is given by equation (2).

While the above dynamics allow pricing of derivative claims to proceed, it is often more convenient to identify the dynamics of the risk neutral process, under which pricing claims proceeds as if all traders were risk neutral. Following along lines identical to Proposition 1 of DRS (2004), under the risk neutral measure $Q$ the dynamics of the asset price is distributionally equivalent to

[Q1]

$$
\begin{array}{llc}
S_{i \Delta t} & =S_{(i-1) \Delta t} e^{\tilde{f}_{i \Delta t}(\Delta t)+\sqrt{h_{i \Delta t}} \tilde{J}_{i(\Delta t)} \sqrt{\Delta t}} \\
h_{(i+1) \Delta t} & =\quad H\left(h_{i} \Delta t, \tilde{J}_{i}(\Delta t)+b \rho \sqrt{\Delta t}\right)
\end{array}
$$

where

$$
\begin{aligned}
\tilde{J}_{i}(\Delta t) & =\tilde{X}_{i}^{(0)}+\sum_{j=1}^{\tilde{N}_{i}(\Delta t)} \tilde{X}_{i}^{(j)}(\Delta t) \text { for } i=1,2, \ldots, n \\
\tilde{X}_{i}^{(0)} & \sim N(0,1) \\
\tilde{X}_{i}^{(j)}(\Delta t) & \sim N\left(\bar{\mu}(\Delta t)+b \rho \gamma(\Delta t) \bar{\gamma}(\Delta t) \sqrt{\Delta t}, \bar{\gamma}^{2}(\Delta t)\right) \text { for } j=1,2, \ldots \\
\tilde{N}_{i}(\Delta t) & \sim \operatorname{Poisson}(\tilde{\lambda}(\Delta t)) \\
\text { where } \tilde{\lambda}(\Delta t) & =\lambda \kappa(\Delta t) \Delta t \\
& \tilde{f}_{i \Delta t}(\Delta t)=f_{i \Delta t}(\Delta t)-b \rho \sqrt{h_{i \Delta t}} \Delta t
\end{aligned}
$$

and $f_{i \Delta t}(\Delta t)$ is defined by equation (14).

Under measure $Q$, the overall dynamics of the asset price is similar in form to the dynamics under the physical measure, P. In particular, the logarithmic return is still a random Poisson 
sum of normal random variables. However, under measure Q, the mean of each of the normal random variables is shifted. Similarly, the sequence of independent Poisson random variables under measure $\mathrm{P}$, are still independent Poisson variables under measure $\mathrm{Q}$ but with a shifted parameter.

Notice from the return equations of the asset under measures $P$ and $Q$ that

$$
\bar{J}_{i}(\Delta t)=\tilde{J}_{i}(\Delta t)+b \rho \sqrt{\Delta t} .
$$

The expected value, $E^{\mathrm{Q}}\left(\tilde{J}_{i}(\Delta t) \mid \mathcal{F}_{(i-1) \Delta t}\right)$, and variance, $\operatorname{Var}^{\mathrm{Q}}\left(\tilde{J}_{i}(\Delta t) \mid \mathcal{F}_{(i-1) \Delta t}\right)$, of $\tilde{J}_{i}(\Delta t)$ are:

$$
\begin{aligned}
E^{\mathrm{Q}}\left(\tilde{J}_{i}(\Delta t)\right) & =\tilde{\lambda}(\Delta t)(\bar{\mu}(\Delta t)+b \rho \gamma(\Delta t) \bar{\gamma}(\Delta t) \sqrt{\Delta t}) \\
\operatorname{Var}^{\mathrm{Q}}\left(\tilde{J}_{i}(\Delta t)\right) & =1+\tilde{\lambda}(\Delta t) \tilde{\gamma}^{2}(\Delta t)
\end{aligned}
$$

where $\tilde{\gamma}^{2}(\Delta t)=(\bar{\mu}(\Delta t)+b \rho \gamma(\Delta t) \bar{\gamma}(\Delta t) \sqrt{\Delta t})^{2}+\bar{\gamma}^{2}(\Delta t)$.

\section{Limiting Forms of the GARCH-Jump Process}

In our model, over each time increment, $\Delta t$, the logarithmic return is a draw from a normal distribution together with a Poisson mixture of normal random variables. As $\Delta t$ gets smaller and smaller, the draws consists of a single normal random variable plus an occasional drawing from another normal distribution. The likelihood of any additional draws will go to zero at a rate faster than $\Delta t$. The limiting behavior of the stock price will obviously depend on the structure of the parameters of the normal innovations as functions of the time increments and upon the specification of the predictable updating process, $H(\cdot)$, for the scaling factor, $h_{i \Delta t}$. Although one can obtain limiting models without jumps, $(\lambda=0)$ this is not of particular interest to us here because such limiting models have already been shown in the literature to arise as limits of standard GARCH-Normal models. In this section we consider two cases for limiting processes that differ according to the specifications for the means and variances as functions of time for

the $X_{i}$ and $\bar{X}_{i}$ variables and the updating process for the scaling factor. The first limiting model has price that follows a jump-diffusion process, while volatility follows a jump process. The second limiting process has jump-diffusive terms in both prices and volatilities and is based on a different updating scheme. We will provide the specific GARCH-Jump models and their corresponding limiting forms under both measures $\mathrm{P}$ and $\mathrm{Q}$.

\subsection{Case 1: Jump-Diffusion Prices with Jumps in Volatility}

In $[P 1]$, let

$$
\begin{array}{ll}
\mu(\Delta t)=\mu / \sqrt{\Delta t} & \gamma^{2}(\Delta t)=\gamma^{2} / \Delta t \\
\bar{\mu}(\Delta t)=\bar{\mu} / \sqrt{\Delta t} & \bar{\gamma}^{2}(\Delta t)=\bar{\gamma}^{2} / \Delta t .
\end{array}
$$


Further assume that the volatility updating scheme, $H(\cdot)$, is of the form:

$$
h_{(i+1) \Delta t}-h_{i \Delta t}=\beta_{0} \Delta t+h_{i \Delta t}\left(\beta_{1}-1\right) \Delta t+\beta_{2} h_{i \Delta t}\left(\frac{\bar{J}_{i}(\Delta t)-E^{\mathrm{P}}\left(\bar{J}_{i}(\Delta t)\right)}{\sqrt{\operatorname{Var}^{\mathrm{P}}\left(\bar{J}_{i}(\Delta t)\right)}}-c\right)^{2} \Delta t .
$$

Proposition 1 below shows the limiting system of the approximating GARCH-Jump model under this specification and with respect to the physical measure $P$. Towards this goal, let $W_{t}$ be a Wiener process, $\pi_{t}$ a Poisson process with intensity $\lambda$, and let $Z_{i}$ 's represent a sequence of independent standard normal random variables that are independent of $W_{t}$ and $\pi_{t}$. We then have:

\section{Proposition 1}

Fix the initial state of the system at $S_{0}$ and $h_{0}$. The GARCH-Jump process, corresponding to system [P1] under the volatility updating equation (23) and parameterization given by equations (21) and (22), weakly converges to (as $\Delta t \rightarrow 0$ for $0 \leq t \leq T$ ):

$$
\begin{aligned}
d l n S_{t} & =f_{t_{-}} d t+\sqrt{h_{t_{-}}} d W_{t}+\left(\bar{\gamma} Z_{\pi_{t}}+\bar{\mu}\right) \sqrt{h_{t_{-}}} d \pi_{t} \\
d h_{t} & =\left(\beta_{0}+\psi_{1} h_{t_{-}}\right) d t+\psi_{2} h_{t_{-}}\left(\bar{\gamma} Z_{\pi_{t}}+\bar{\mu}\right)^{2} d \pi_{t}
\end{aligned}
$$

where

$$
\begin{aligned}
\psi_{1} & =\beta_{1}+\psi_{2}\left(1+c^{2}\left(1+\lambda \hat{\gamma}^{2}\right)\right)-1 \\
\psi_{2} & =\beta_{2} /\left(1+\lambda \hat{\gamma}^{2}\right) \\
f_{t} & =r-\frac{h_{t}}{2}-\sqrt{h_{t}} b \rho+\lambda \kappa\left(1-\exp \left(\sqrt{h_{t}}(\bar{\mu}+b \rho \gamma \bar{\gamma})+\frac{1}{2} h_{t} \bar{\gamma}^{2}\right)\right) .
\end{aligned}
$$

Proof: See Appendix.

First note that setting $\Delta t=1$ for this GARCH-Jump process gives rise to the benchmark discrete-time GARCH-Jump model studied by DRS (2004). The limiting model has discontinuous stock price and volatility paths. Notice that when $\psi_{2}=0$, the scaling factor, $h_{t}$ is deterministic, and with a further restriction of $\psi_{1}=0$, a simple constant-volatility jump-diffusion model obtains. Thus, the jump-diffusion model of Merton (1976) is nested in this family. In our model both intensity risk and jump magnitude risk are priced and the notion that jumps can only occur in returns, but not in volatilities, is removed. When $\psi_{2}$ is released from 0 then the volatility process is no longer continuous. In this case, the drift of volatility is influenced by the continuous innovations in the asset prices. Further, when jumps occur in returns, they are accompanied by jumps in volatility.

We now consider the process under the risk neutral measure, $Q$. Let $\tilde{W}_{t}$ and $\tilde{B}_{t}$ be two independent Wiener processes, $\tilde{\pi}_{t}$ a Poisson process with intensity $\tilde{\lambda}$, and let $\tilde{Z}_{i}$ 's represent a sequence of independent standard random variables that are independent of $\tilde{W}_{t}, \tilde{B}_{t}$ and $\tilde{\pi}_{t}$. We have: 


\section{Proposition 2}

Fix the initial state of the system at $S_{0}$ and $h_{0}$. The risk neutral GARCH-Jump process, corresponding to system [Q1] under the volatility updating equation (23) and parameterization given by equations (21) and (22), weakly converges to (as $\Delta t \rightarrow 0$ for $0 \leq t \leq T$ ):

$$
\begin{aligned}
d l n S_{t} & =\tilde{f}_{t_{-}} d t+\sqrt{h_{t_{-}}} d \tilde{W}_{t}+\left(\bar{\gamma} \tilde{Z}_{\pi_{t}}+\bar{\mu}+b \rho \gamma \bar{\gamma}\right) \sqrt{h_{t_{-}}} d \tilde{\pi}_{t} \\
d h_{t} & =\left(\beta_{0}+\psi_{1} h_{t_{-}}\right) d t+\psi_{2} h_{t_{-}}\left(\bar{\gamma} \tilde{Z}_{\pi_{t}}+\bar{\mu}+b \rho \gamma \bar{\gamma}\right)^{2} d \tilde{\pi}_{t}
\end{aligned}
$$

where

$$
\tilde{f}_{t}=r-\frac{h_{t}}{2}+\lambda \kappa\left(1-\exp \left(\sqrt{h_{t}}(\bar{\mu}+b \rho \gamma \bar{\gamma})+\frac{1}{2} h_{t} \bar{\gamma}^{2}\right)\right)
$$

and $\psi_{1}$ and $\psi_{2}$ have been defined in Proposition 1.

Proof: See Appendix.

Proposition 2 shows that the overall behavior of the limiting processes under both measures take on a similar form, with adjustments to the drift, intensity and to the magnitude of the jumps, that reflect the risk premiums under measure $P$.

We can explore the convergence behavior of option prices generated by the GARCH-Jump model as the time increment is refined. The convergence pattern will be investigated later in Section 3 .

\subsection{Case 2: Jump-Diffusions in both Prices and Volatilities}

The exact nature of the limiting models depends on our specification of the predictable process for the scaling factor, $h_{i \Delta t}$. In Case 1, we used an NGARCH specification. As a result, given that a jump occurs, its size is directly proportional to $h_{i \Delta t}$. Models can readily be obtained where the effects of jumps are not proportional to the level of the scaling factor. As an example, we begin by replacing the NGARCH process, given in equation (10) with the following discrete-time threshold GARCH (TGARCH) model:

$$
\begin{aligned}
& \phi_{t}=\beta_{0}+\beta_{1} \phi_{t-1}+\beta_{2}\left|\frac{\bar{J}_{t-1}-\lambda \bar{\mu}}{\sqrt{1+\lambda \hat{\gamma}^{2}}}\right|+\beta_{3} \max \left(-\frac{\bar{J}_{t-1}-\lambda \bar{\mu}}{\sqrt{1+\lambda \hat{\gamma}^{2}}}, 0\right) \\
& h_{t}=\phi_{t}^{2} .
\end{aligned}
$$

Different limiting processes are expected. For example, we could specify an approximating process for $\phi_{t}$, over time increment $\Delta t$, of the form:

$$
\begin{aligned}
\phi_{(i+1) \Delta t}-\phi_{i \Delta t}= & \left(\beta_{0}+\beta_{2} q_{2}+\beta_{3} q_{3}\right) \Delta t+\phi_{i \Delta t}\left(\beta_{1}-1\right) \Delta t \\
& +\beta_{2}\left[\left|\frac{\bar{J}_{i}(\Delta t)-E^{\mathrm{P}}\left(\bar{J}_{i}(\Delta t)\right)}{\sqrt{\operatorname{Var} \mathrm{P}\left(\bar{J}_{i}(\Delta t)\right)}}\right|-q_{2}\right] \sqrt{\Delta t}
\end{aligned}
$$




$$
+\beta_{3}\left[\max \left(-\frac{\bar{J}_{i}(\Delta t)-E^{\mathrm{P}}\left(\tilde{J}_{i}(\Delta t)\right)}{\sqrt{\operatorname{Var}^{\mathrm{P}}\left(\tilde{J}_{i}(\Delta t)\right)}}, 0\right)-q_{3}\right] \sqrt{\Delta t}
$$

where $q_{2}=E^{P}\left|\frac{\bar{X}_{i}^{(0)}}{\sqrt{\operatorname{Var}^{P}\left(\bar{J}_{i}(\Delta t)\right)}}\right|$ and $q_{3}=E^{P}\left[\max \left(-\frac{\bar{X}_{i}^{(0)}}{\sqrt{\operatorname{Var}^{P}\left(\bar{J}_{i}(\Delta t)\right)}}\right), 0\right]$.

To obtain a specific model, we need to specify the structure of the parameters of the normal innovations as functions of the time increments. If we assume the parameterization as in Case 1 , the price process naturally becomes a jump-diffusion process similar to that in Proposition 1. In particular for this structure we have:

\section{Proposition 3}

Fix the initial state of the system at $S_{0}$ and $h_{0}$. The GARCH-Jump process, corresponding to system [P1] under the volatility updating equation (30) and parameterization given by equations (21) and (22), weakly converges to (as $\Delta t \rightarrow 0$ for $0 \leq t \leq T$ ):

$$
\begin{aligned}
d l n S_{t}= & f_{t_{-}} d t+\sqrt{h_{t_{-}}} d W_{t}+\left(\bar{\gamma} Z_{\pi_{t}}+\bar{\mu}\right) \sqrt{h_{t_{-}}} d \pi_{t} \\
d \phi_{t}= & \left(\beta_{0}+\beta_{2} q_{2}+\beta_{3}\left(q_{3}+\frac{\lambda \bar{\mu}}{2 \sqrt{1+\lambda \hat{\gamma}^{2}}}\right)+\left(\beta_{1}-1\right) \phi_{t_{-}}\right) d t \\
& -\frac{\beta_{3}}{2 \sqrt{1+\lambda \hat{\gamma}^{2}}} d W_{t}+\left(\beta_{2}+\frac{\beta_{3}}{2} \sqrt{1+\lambda \hat{\gamma}^{2}}\right) \sqrt{\frac{\pi-2}{\pi}} d B_{t} \\
& +\frac{1}{\sqrt{1+\lambda \hat{\gamma}^{2}}}\left[\beta_{2}\left|\bar{\gamma} Z_{\pi_{t}}+\bar{\mu}\right|+\beta_{3} \max \left(-\bar{\gamma} Z_{\pi_{t}}-\bar{\mu}, 0\right)\right] d \pi_{t} \\
h_{t}= & \phi_{t}^{2}
\end{aligned}
$$

where

$$
f_{t}=r-\frac{h_{t}}{2}-\sqrt{h_{t}} b \rho+\lambda \kappa\left(1-\exp \left(\sqrt{h_{t}}(\bar{\mu}+b \rho \gamma \bar{\gamma})+\frac{1}{2} h_{t} \bar{\gamma}^{2}\right)\right)
$$

Proof: See Appendix.

As a special case, the limiting volatility process, obtained by applying Ito's lemma and letting $\beta_{0}+\beta_{2} q_{2}+\beta_{3}\left(q_{3}+\frac{\lambda \bar{\mu}}{2 \sqrt{\left.1+\lambda \hat{\gamma}^{2}\right)}}\right)=0$, is given by:

$$
\begin{aligned}
d h_{t}= & {\left[\frac{\beta_{3}^{2}}{4\left(1+\lambda \hat{\gamma}^{2}\right)}+\left(\beta_{2}+\frac{\beta_{3}}{2}\right)^{2} \frac{\pi-2}{\pi\left(1+\lambda \hat{\gamma}^{2}\right)}+2\left(\beta_{1}-1\right) h_{t_{-}}\right] d t } \\
& -\frac{\beta_{3}}{\sqrt{1+\lambda \hat{\gamma}^{2}}} \sqrt{h_{t_{-}}} d W_{t}+\left(2 \beta_{2}+\beta_{3}\right) \sqrt{\frac{\pi-2}{\pi\left(1+\lambda \hat{\gamma}^{2}\right)}} \sqrt{h_{t_{-}}} d B_{t} \\
& +\frac{1}{1+\lambda \hat{\gamma}^{2}}\left[\beta_{2}\left|\bar{\gamma} Z_{\pi_{t}}+\bar{\mu}\right|+\beta_{3} \max \left(-\bar{\gamma} Z_{\pi_{t}}-\bar{\mu}, 0\right)\right]^{2} d \pi_{t} .
\end{aligned}
$$

In contrast to the limiting model in Proposition 1, this limiting form allows for jump-diffusion in both price and volatility. This model is a mean-reverting square root process with jumps for 
$h_{t}$. By turning off jumps, the limiting model nests the square root stochastic volatility model given in Scott (1987) and Heston (1993). Without switching off jumps, the volatility dynamic in equation (34) is more general than that in Bakshi, Cao and Chen (1997), Bates (2000) or Pan (2002), for it allows for volatility jumps as well. ${ }^{5}$

Finally, we consider the dynamics under the risk neutral measure. Let $\tilde{W}_{t}$ and $\tilde{B}_{t}$ be two independent Wiener processes under measure $\mathrm{Q}$. Under the same measure, let $\tilde{\pi}_{t}$ denote a Poisson process with intensity $\lambda \kappa$ that is independent of $\tilde{W}_{t}$ and $\tilde{B}_{t}$, and let $\tilde{Z}_{i}$ 's represent a sequence of independent standard normal random variables that are independent of $\tilde{W}_{t}, \tilde{B}_{t}$ and $\tilde{\pi}_{t}$.

\section{Proposition 4}

Fix the initial state of the system at $S_{0}$ and $h_{0}$. The risk neutral GARCH-Jump process, corresponding to system [Q1] under the volatility updating equation (30) and parameterization given by equations (21) and (22), weakly converges to (as $\Delta t \rightarrow 0$ for $0 \leq t \leq T$ ):

$$
\begin{aligned}
d l n S_{t}= & \tilde{f}_{t_{-}} d t+\sqrt{h_{t_{-}}} d \tilde{W}_{t}+\left(\bar{\gamma} \tilde{Z}_{\pi_{t}}+\bar{\mu}+b \rho \gamma \bar{\gamma}\right) \sqrt{h_{t_{-}}} d \tilde{\pi}_{t} \\
d \phi_{t}= & \left(\beta_{0}+\beta_{2} q_{2}+\beta_{3}\left(q_{3}+\frac{\lambda \bar{\mu}-b \rho}{2 \sqrt{\left.1+\lambda \hat{\gamma}^{2}\right)}}\right)+\left(\beta_{1}-1\right) \phi_{t_{-}}\right) d t \\
& -\frac{\beta_{3}}{2 \sqrt{1+\lambda \hat{\gamma}^{2}}} d \tilde{W}_{t}+\left(\beta_{2}+\frac{\beta_{3}}{2}\right) \sqrt{\frac{\pi-2}{\pi\left(1+\lambda \hat{\gamma}^{2}\right)}} d \tilde{B}_{t} \\
& +\frac{1}{\sqrt{1+\lambda \hat{\gamma}^{2}}}\left[\beta_{2}\left|\bar{\gamma} \tilde{Z}_{\pi_{t}}+\bar{\mu}+b \rho \gamma \bar{\gamma}\right|+\beta_{3} \max \left(-\bar{\gamma} \tilde{Z}_{\pi_{t}}-\bar{\mu}-b \rho \gamma \bar{\gamma}, 0\right)\right] d \tilde{\pi}_{t} \\
h_{t}= & \phi_{t}^{2}
\end{aligned}
$$

where $\tilde{f}_{t}=r-\frac{h_{t}}{2}+\lambda \kappa\left[1-\exp \left(\sqrt{h_{t}}(\bar{\mu}+b \rho \gamma \bar{\gamma})+\frac{1}{2} h_{t} \bar{\gamma}^{2}\right)\right]$.

Proof: See Appendix.

\section{Convergence Speeds of Option Prices Using Simulation}

In this section we examine convergence issues of GARCH-Jump option prices to their continuous time limits. We assume that the true risk neutral processes are given by Proposition 2 for our Case 1, and by Proposition 4, for Case 2. European claims can be priced directly off the continuous time processes using Monte Carlo methods augmented with variance reduction techniques. The underlying dynamics are typically approximated by the Euler scheme. Our objective is to compare convergence pattern of the Euler scheme with that of the scheme built around the approximating GARCH-Jump process. Because we are comparing the option prices

\footnotetext{
${ }^{5}$ In our model, the same jump affects both price and volatility. If one wants to switch off just one of them, two separate jump sources need to be built into the approximating model.
} 
which are essentially expected values, we in effect deal with the weak order of convergence for these approximation schemes. Interestingly, the Euler scheme is known to have a weak convergence order of 1 for diffusion models even though it only has a strong convergence order of 1/2 (see Kloeden and Platen (1992)). For jump-diffusion models, the Euler scheme continues to have a weak convergence order of 1, a result established in Theorem 5.2 of Maghsoodi (1998).

For both Cases 1 and 2, we take the limiting jump-diffusion processes and very carefully simulate a set of "true" option prices. The set consists of various strikes surrounding the 30 day at-the-money contract. The simulations are conducted using 50,000 sample paths and an Euler scheme with a partition of $2^{10}=1024$ time steps per day, or equivalently a time partition of about 84 seconds. In addition we use antithetic variance reduction techniques. The resulting set of true option prices are stored.

We next pick a series of time partitions and use the Euler scheme to establish benchmark rate of convergence to the true prices. Finally, we compute the same set of option prices using our GARCH-Jump approximating scheme using the same time partitions. Since large sample sizes were used in the simulations, the standard errors of the estimates were generally smaller than one cent, and so the confidence intervals are not reported. The percentage errors in the pricing are documented for the two approximating schemes and for each time partition. As the time increments are refined, the two sets of approximating option prices should converge to their true common values. In this section we are interested in documenting the relative speed of convergence.

To establish a set of parameters, we used the time series of the $S \& P 500$ index values to estimate the relevant parameters of equation (23) or (30), depending on the case. Both estimations were conducted by setting $\Delta t=1$ day. Since not all the parameters can be fully identified from the time series alone, we estimated the models under the restriction that $\kappa=1$ and $\gamma=1$. The maximum likelihood estimation method is detailed in DRS (2004). ${ }^{6}$ In a nutshell, the likelihood function of our GARCH-Jump model can be explicitly expressed in a simple analytical form just like the standard GARCH model. Maximum likelihood estimation can then be carried out using a standard gradient-based optimization method.

For Case 1 where the volatility updating scheme is an NGARCH model, our remaining benchmark parameter estimates (standard errors) are:

$\begin{array}{llllllll}\beta_{0} & \beta_{1} & \beta_{2} & c & b \rho & \lambda & \bar{\gamma} & \bar{\mu} \\ 1.65 \mathrm{e}-7 & 0.844 & 0.0756 & 0.7714 & -0.0723 & 2.20 & 2.096 & 0.0332 \\ (0.00663) & (0.0062) & (0.0041) & (0.0008) & (0.0161) & (0.0004) & (0.0014) & (0.0161)\end{array}$

For Case 2, the maximum likelihood estimates (standard errors) are:

\footnotetext{
${ }^{6}$ DRS (2004) use panel of option prices in addition to time series data to estimate the parameters and to evaluate the effectiveness of specific discrete time models, both from the point of view of explaining the volatility smile and the ability to hedge over finite periods.
} 


$\begin{array}{llllllll}\beta_{0} & \beta_{1} & \beta_{2} & \beta_{3} & b \rho & \lambda & \bar{\gamma} & \bar{\mu} \\ 2.3538 \mathrm{e}-5 & 0.95653 & 4.1551 \mathrm{e}-6 & 0.000345 & -0.0278 & 1.1478 & 1.2837 & 0.04760 \\ (0.041) & (0.0001) & (0.0001) & (0.00001) & (0.002) & (0.001) & (0.0021) & (0.0002)\end{array}$

In addition, for our experiments we assume $r=5 \%$ per year, $S_{0}=500$, and the initial volatility was $30 \%$ annualized. The time partitions start at one per day, two per day, four per day, and keep doubling until we reach 1024 partitions per day.

Table 1 shows the percentage errors for both approximating methods over a full range of strike prices for the 30- day contracts. As can be seen, the approximating GARCH-Jump process produces consistently more accurate results except for the time partitions that are close to 1024. Note that we have set the Euler approximation prices with a time partition of 1024 as the "true" price. Needless to say, the results are "engineered" to be in favor of the Euler scheme when the time partition is close to 1024.

Table 1 Here

Note that the GARCH approximating scheme produces errors of magnitude less than one percent for 256 partitions. Generally, this error is less than two cents and is of the order of simulation error.

In order to gain a better understanding of the convergence behavior, we conduct an analysis on the absolute difference between the approximating option price and the true price by relating it to the number of time steps. In particular, the absolute difference can be approximated by:

$$
\left|C(n)-C_{0}\right| \approx a n^{-\delta} .
$$

Let $n=2^{k}$ and restating the above expression in terms of $k$ we obtain:

$$
\left|C\left(2^{k}\right)-C_{0}\right| \approx a 2^{-k \delta} .
$$

Therefore,

$$
\ln \left(\left|C\left(2^{k}\right)-C_{0}\right|\right) \approx \ln (a)-k \delta \ln 2 .
$$

A plot of the logarithmic absolute price difference in relation to $k$ thus reveals the convergence behavior. A smaller intercept implies a smaller convergence constant, i.e., a better numerical scheme when $k$ is small, whereas a steeper slope suggests a faster convergence rate, i.e., a better numerical scheme when $k$ is large.

Figure 1 shows how the option prices under Case 1 converge under the Euler and the GARCHJump approximation schemes. The plots on the left-hand side reveal graphically the results in Table 1. The plots on the right-hand side, however, indicate that the GARCH approximating process has a smaller convergence constant and is competitive with the Euler scheme in terms of the convergence rate. These convergence plots are merely suggestive, however. As noted earlier, the Monte Carlo errors of the computed option prices are typically less than one cent. The same magnitude of Monte Carlo error will have a much larger impact on the logarithmic absolute pricing error for the points corresponding to larger $k$ 's vis-a-vis the points corresponding to smaller $k$ 's.

Figure 1 Here 
We now turn to Case 2 and repeat the above analysis. Figure 2 and Table 2 summarize the overall convergence results and the general nature is fairly similar to Case 1 except that the improvement over the Euler scheme in terms of the convergence constant is not as dramatic.

Table 2 and Figure 2 Here

Within the class of option models nested by Case 2 are many important models for which analytical solutions for European options exist. However, there also are an enormous number of potentially useful models outside the family of models for which analytical solutions exist. For such models, our results provide an alternative to Euler approximations as a mechanism for more efficiently computing option prices.

We experimented with other sets of parameters and the results seem fairly stable. In particular, with $\Delta t=1$ or 0.5 days, option pricing errors under approximating GARCH processes appears to be consistently smaller than errors generated by Euler approximations.

Even if one prefers to begin with modeling prices and volatilities by a bivariate process in continuous time, as above, there are significant advantages in using the GARCH-Jump model as an approximating device. In fact, the above numerical results indicate that the approximating GARCH-Jump model provides a more efficient way of computing option prices than the use of the Euler approximation scheme.

\section{Other Limiting Models}

Clearly, the same methodology can be applied to different volatility updating functions, $H(\cdot)$, to obtain different limiting jump diffusion models. It also is possible to allow the functional dependence on time in the means and variances of the $X$ and $\bar{X}$ variables to be of different forms. For example we could retain an NGARCH structure, as in Case 1, but allow the means and variances of the $X$ and $\bar{X}$ to be of the form:

$$
\begin{array}{ll}
\mu(\Delta t)=\mu / \Delta t^{1 / 4} & \gamma^{2}(\Delta t)=\gamma^{2} / \sqrt{\Delta t} \\
\bar{\mu}(\Delta t)=\bar{\mu} / \Delta t^{1 / 4} & \bar{\gamma}^{2}(\Delta t)=\bar{\gamma}^{2} / \sqrt{\Delta t} .
\end{array}
$$

In contrast to Case 1, where the limiting price follows a jump diffusion and volatility follows a jump process, this parameterization leads to a limiting process where the price follows a diffusion, and volatility follows a jump-diffusion.

To see this we modify the updating equation (23) to the form:

$$
\begin{aligned}
h_{(i+1) \Delta t}-h_{i \Delta t} & =\beta_{0} \Delta t+h_{i \Delta t}\left(\beta_{1}+\beta_{2}\left(1+c^{2}\right)-1\right) \Delta t \\
& +\beta_{2} h_{i \Delta t}\left[\left(\frac{\bar{J}_{i}(\Delta t)-E^{\mathrm{P}}\left(\bar{J}_{i}(\Delta t)\right)}{\left.\left.\sqrt{\operatorname{Var} \mathrm{P}\left(\bar{J}_{i}(\Delta t)\right)}-c\right)^{2}-\left(1+c^{2}\right)\right] \sqrt{\Delta t}} .\right.\right.
\end{aligned}
$$

Notice that for $\Delta t=1$, this scheme is identical to the NGARCH scheme in Case 1. For this specification, we have: 


\section{Proposition 5}

Fix the initial state of the system at $S_{0}$ and $h_{0}$. The GARCH-Jump process, corresponding to system [P1] with volatility updating scheme provided by equation (40) and parameterization as given by equations (38) and (39), weakly converges to (as $\Delta t \rightarrow 0$ for $0 \leq t \leq T$ ):

$$
\begin{aligned}
d l n S_{t}= & f_{t_{-}} d t+\sqrt{h_{t_{-}}} d W_{t} \\
d h_{t}= & \left\{\beta_{0}+h_{t_{-}}\left[\beta_{1}+\beta_{2}\left(1+c^{2}-\lambda \hat{\gamma}^{2}\right)-1\right]\right\} d t-2 c \beta_{2} h_{t_{-}} d W_{t}+\sqrt{2} \beta_{2} h_{t_{-}} d B_{t} \\
& +\beta_{2} h_{t_{-}}\left(\bar{\gamma} Z_{\pi_{t}}+\bar{\mu}\right)^{2} d \pi_{t}
\end{aligned}
$$

where $f_{t}=r-\frac{h_{t}}{2}-\sqrt{h_{t}}$ b and $\hat{\gamma}^{2}=\bar{\mu}^{2}+\bar{\gamma}^{2}$.

Proof: See Appendix.

This limiting model has continuous asset price paths but discontinuous volatility paths. Unlike our previous case where the local scaling factor, $h_{t}$, was not the local variance, in this model the local scaling factor, $h_{t}$ does become the local variance. In this model $\beta_{2}$ plays an important role in determining jump and diffusion effects in volatility and the correlation between volatility and return.

For completeness, we consider the corresponding process under the risk neutral measure, $Q$. Although the limiting return dynamic under measure $Q$ follows in a way similar to its corresponding part under measure $\mathrm{P}$, the limiting volatility process requires additional work because more of the parameters are functions of $\Delta t$.

As before, let $\tilde{W}_{t}$ and $\tilde{B}_{t}$ be two independent Wiener processes, $\tilde{\pi}_{t}$ a Poisson process with intensity $\tilde{\lambda}$, and let $\tilde{Z}_{i}$ 's represent a sequence of independent standard random variables that are independent of $\tilde{W}_{t}, \tilde{B}_{t}$ and $\tilde{\pi}_{t}$.

\section{Proposition 6}

Fix the initial state of the system at $S_{0}$ and $h_{0}$. The risk neutral GARCH-Jump process, corresponding to system [Q1] with volatility updating scheme provided by equation (40) and parameterization as given by equations (38) and (39), weakly converges to (as $\Delta t \rightarrow 0$ for $0 \leq t \leq T$ ):

$$
\begin{aligned}
d l n S_{t}= & \tilde{f}_{t_{-}} d t+\sqrt{h_{t_{-}}} d \tilde{W}_{t} \\
d h_{t}= & \left\{\beta_{0}+h_{t_{-}}\left[\beta_{1}+\beta_{2}\left(1+c^{2}-\lambda \hat{\gamma}^{2}-2 c b \rho\right)-1\right]\right\} d t \\
& -2 c \beta_{2} h_{t_{-}} d \tilde{W}_{t}+\sqrt{2} \beta_{2} h_{t_{-}} d \tilde{B}_{t}+\beta_{2} h_{t_{-}}\left(\bar{\gamma} \tilde{Z}_{\pi_{t}}+\bar{\mu}\right)^{2} d \tilde{\pi}_{t}
\end{aligned}
$$

where $\hat{\gamma}^{2}=\bar{\mu}^{2}+\bar{\gamma}^{2}$ and $\tilde{f}_{t}=r-\frac{h_{t}}{2}$.

Proof: See Appendix.

This example shows that the limiting form of the NGARCH-Jump model is not unique. Comparing Case 1 with this example demonstrates that by altering the GARCH coefficients (as functions of $\Delta t$ ), one can obtain different limiting models. In fact, a deterministic volatility jump-diffusion model can also be obtained in a way similar to Corradi (2000). It is informative to know such a possibility exists, but degenerate limits are not as constructive as the non-degenerate limits presented in our paper.

The convergence rate for this example is not expected to be high because the terms $(\Delta t)^{0.25}$ appear in the approximating GARCH-Jump model. Indeed, computational experiments confirm its slow 
convergence.

If the objective is to develop efficient algorithms for pricing options under the risk neutral measure then it should be recognized that there is no real need to link the dynamics of the physical process with the risk neutral process. This being the case, there is no need to curtail $f(\cdot)$ as we have done. Indeed, if the goal is just to price options with a flexible model, then one can begin directly with the dynamics under the Q-measure. One advantage of this approach is that the function $f(\cdot)$ is therefore less constrained. For example, we could specify the drift term as being the drift term in the continuous limit process. The above propositions which link GARCH models to continuous time limiting models, therefore would continue to hold, but for a larger class of $f(\cdot)$ functions.

\section{Conclusion}

In this paper we have extended Nelson (1990) and Duan (1997) by considering limiting models for the GARCH-Jump process. These limiting models can have diffusive prices and volatilities as well as random correlated jumps in either or both processes.

In addition to establishing the dynamics under the physical probability measure, we also identified the risk-neutral dynamics. The resulting limiting models are interesting in their own right, converge rapidly to their continuous-time counterparts in comparison to the Euler approximation scheme, and allow us to relate discrete time GARCH-Jump models to the large literature on stochastic volatility and jumps.

The approximating GARCH-Jump model for options contains as special limiting cases, jump-diffusion models, like Merton (1976), and diffusive stochastic volatility models, like Heston (1993) or Hull and White (1987). In addition, limiting cases of the model that allow for jumps in both prices and volatilities can be constructed that allow for option pricing along the lines of Duffie, Pan and Singleton (2001).

Of course, a disadvantage of the GARCH-Jump models is that they rely, for the most part, on Monte Carlo methods for pricing. In contrast, as long as one remains in the class of affine models, pseudo analytical solutions for European options exist. To date, however, many problems with affine models have been identified, and it is possible that we may have to search outside this family. Further, even if one remains in the affine class, simulation techniques may have to be invoked for pricing American options. So even within the class of affine models, the GARCH approximating processes may prove useful for establishing efficient numerical schemes for pricing claims.

Finally, our results related to a local volatility updating equation of the form based either on an NGARCH or TGARCH updating scheme. Härdle and Hafner (2000) have investigated the TGARCH volatility updating mechanism and using simulations, they conclude that this scheme might be preferable. Since our theory is not limited to these two particular updating schemes, other specifications could be considered. It remains for future empirical research to evaluate alternative jump-diffusion models for prices and volatilities using the approximating GARCH models outlined here. 


\section{References}

Bakshi, G., C. Cao and Z. Chen (1997) Empirical Performance of Alternative Option Pricing Models, Journal of Finance 53, 499-547.

Bates, D. (2000) Post-'87 Crash Fears in S\&P 500 Futures Options, Journal of Econometrics 94, 181-238.

Corradi V. (2000) Reconsidering the Continuous Time Limit of the GARCH(1,1) Process, Journal of Econometrics 96, 145-153.

Duan, J. (1995) The GARCH Option Pricing Model, Mathematical Finance 5, 13-32.

Duan, J. (1997) Augmented GARCH(p,q) Process and its Diffusion Limit, Journal of Econometrics 79, $97-127$.

Duan, J., P. Ritchken and Z. Sun (2004) Jump Starting GARCH: Pricing and Hedging Options with Jumps in Returns and Volatilities, Working Paper University of Toronto and Case Western Reserve University.

Duan, J., and J.-G. Simonato (2001) American Option Pricing under GARCH by a Markov Chain Approximation, Journal of Economic Dynamics and Control 25, 1689-1718.

Duffie, D., K. Singleton and J. Pan (1999) Transform Analysis and Asset pricing for Affine Jump Diffusions, Econometrica, 68, 1343-1376.

Eraker B., M. Johannes and N. Polson (2003) The Impact of Jumps in Volatility and Returns, Journal of Finance 3, 1269-1300.

Härdle W., and C. Hafner (2000) Discrete Time Option Pricing with Flexible Volatility Estimation, Finance and Stochastics 4, 189-207.

Heston, S. (1993) A Closed-Form Solution for Options with Stochastic Volatility, Review of Financial Studies 6, 327-344.

Heston, S., and S. Nandi (2000) A Closed Form GARCH Option Pricing Model, Review of Financial Studies 13, 585-625.

Hull, J. and A. White (1987) The Pricing of Options on Assets with Stochastic Volatility, Journal of Finance 42, 281-300.

Jacquier, E., N. Polson, and P. Rossi (1994) Bayesian Analysis of Stochastic Volatility Models, Journal of Business and Economic Statistics 12, 1-19.

Kloeden, P. and E. Platen (1992), Numerical Solution of Stochastic Differential Equations, SpringerVerlag, Berlin.

Kurtz, T. and P. Protter (1991) Weak Limit Theorems for Stochastic Integrals and Stochastic Differential Equations, Annals of Probability 19, 1035-1070.

Maghsoodi, Y. (1998) Exact Solutions and Doubly Efficient Approximations of Jump-Diffusion Ito Equations, Stochastic Analysis and Applications 16, 1049-1072.

Merton, R. (1976) Option Pricing when the Underlying Stock Returns are Discontinuous, Journal of Financial Economics 3, 125-144. 
Naik, V. and M. Lee (1990) General Equilibrium Pricing of Options on the Market Portfolio with Discontinuous Returns, Review of Financial Studies 3, 493-521.

Nelson, D. (1990) ARCH Models as Diffusion Approximations, Journal of Econometrics 45, 7-38

Pan, J. (2002) The Jump-risk Premia Implicit in Options: Evidence from an Integrated Time-series Study, Journal of Financial Economics 63, 3-50.

Renault, E. (1997) Econometric Models of Option Pricing Errors, Advances in Economics and Econometrics: Theory and Applications 3 (Kreps, D. and K. Wallis, eds), Cambridge University Press, 223-278.

Ritchken, P. and R. Trevor (1999) Pricing Options Under Generalized GARCH and Stochastic Volatility Processes, Journal of Finance 54, 377-402.

Singleton, K. (2001) Estimation of Affine Asset Pricing Models using the Empirical Characteristic Function, Journal of Econometrics 102, 111-141.

Scott, L. (1987) Option Pricing When the Variance Changes Randomly: Theory, Estimation and An Application, Journal of Financial and Quantitative Analysis 22, 419-438.

Stein, E., and J. Stein (1991) Stock Price Distributions with Stochastic Volatility: An Analytic Approach, Review of Financial Studies 4, 727-752.

Wiggins, J. (1987) Option Values under Stochastic Volatility: Theory and Empirical Evidence, Journal of Financial Economics 19, 351-372. 


\section{Appendix}

\section{Proof of Proposition 1}

The GARCH-Jump model under measure $P$ is

$$
\begin{aligned}
\ln S_{i \Delta t}-\ln S_{(i-1) \Delta t} & =f_{i \Delta t}(\Delta t)+\sqrt{h_{i \Delta t}} \bar{J}_{i}(\Delta t) \sqrt{\Delta t} \\
h_{(i+1) \Delta t}-h_{i \Delta t} & =\beta_{0} \Delta t+h_{i \Delta t}\left(\beta_{1}-1\right) \Delta t+\beta_{2} h_{i \Delta t}\left(\frac{\bar{J}_{i}(\Delta t)-\lambda \bar{\mu} \sqrt{\Delta t}}{\sqrt{1+\lambda \hat{\gamma}^{2}}}-c\right)^{2} \Delta t
\end{aligned}
$$

where $f_{i \Delta t}(\Delta t)$, given by equation (14), becomes

$$
f_{i \Delta t}(\Delta t)=\left\{r-\frac{h_{i \Delta t}}{2}-\sqrt{h_{i \Delta t}} b \rho+\lambda \kappa\left[1-\exp \left(\sqrt{h_{i \Delta t}}(\bar{\mu}+b \rho \gamma \bar{\gamma})+\frac{1}{2} h_{i \Delta t} \bar{\gamma}^{2}\right)\right]\right\} \Delta t .
$$

In the above, $\bar{J}_{i}(\Delta t)=\bar{X}_{i}^{(0)}+\sum_{j=1}^{N_{i}(\Delta t)} \bar{X}_{i}^{(j)}(\Delta t), \bar{X}_{i}^{(0)} \sim N(0,1), \bar{X}_{i}^{(j)}(\Delta t) \sim N\left(\frac{\bar{\mu}}{\sqrt{\Delta t}}, \frac{\bar{\gamma}^{2}}{\Delta t}\right), N_{i}(\Delta t)$ is a Poisson random variable with parameter $\lambda \Delta t$, and $\bar{X}_{i}^{(j)}(j=0,1, \cdots)$ are independent.

It should be understood that we are dealing with the space of functions on $[0, T]$ that are rightcontinuous and have left-hand limits, and this space is endowed with the Skorohod topology.

First, we have the following result for the conditional mean return:

$$
\frac{f_{[n t / T] \Delta t}(\Delta t)}{\Delta t} \longrightarrow r-\frac{h_{t_{-}}}{2}-\sqrt{h_{t_{-}}} b \rho+\lambda \kappa\left[1-\exp \left(\sqrt{h_{t_{-}}}(\bar{\mu}+b \rho \gamma \bar{\gamma})+\frac{1}{2} h_{t_{-}} \bar{\gamma}^{2}\right)\right] .
$$

Next, define

$$
\begin{aligned}
H_{n}^{(1)}(t) & \equiv \sum_{i=1}^{[n t / T]} \bar{J}_{i}(\Delta t) \sqrt{\Delta t} \\
& =\sum_{i=1}^{[n t / T]} \bar{X}_{i}^{(0)} \sqrt{\Delta t}+\sum_{i=1}^{[n t / T]} \sum_{j=1}^{N_{i}(\Delta t)} \bar{X}_{i}^{(j)}(\Delta t) \sqrt{\Delta t}
\end{aligned}
$$

and

$$
\begin{aligned}
H_{n}^{(2)}(t) \equiv & \sum_{i=1}^{[n t / T]}\left(\frac{\bar{J}_{i}(\Delta t)-\lambda \bar{\mu} \sqrt{\Delta t}}{\sqrt{1+\lambda \hat{\gamma}^{2}}}-c\right)^{2} \Delta t \\
= & \sum_{i=1}^{[n t / T]}\left(\frac{\bar{X}_{i}^{(0)}}{\sqrt{1+\lambda \hat{\gamma}^{2}}}-c\right)^{2} \Delta t+2 \sum_{i=1}^{[n t / T]}\left(\frac{\bar{X}_{i}^{(0)}}{\sqrt{1+\lambda \hat{\gamma}^{2}}}-c\right)\left(\frac{\sum_{j=1}^{N_{i}(\Delta t)} \bar{X}_{i}^{(j)}(\Delta t) \Delta t-\lambda \bar{\mu}(\Delta t)^{3 / 2}}{\sqrt{1+\lambda \hat{\gamma}^{2}}}\right) \\
& +\sum_{i=1}^{[n t / T]}\left(\frac{\sum_{j=1}^{N_{i}(\Delta t)} \bar{X}_{i}^{(j)}(\Delta t) \sqrt{\Delta t}-\lambda \bar{\mu} \Delta t}{\sqrt{1+\lambda \hat{\gamma}^{2}}}\right)^{2} .
\end{aligned}
$$

Note first that $\sum_{i=1}^{[n t / T]} \bar{X}_{i}^{(0)} \sqrt{\Delta t}$ in $H_{n}^{(1)}(t)$ converges weakly to the standard Brownian motion $W_{t}$ by Donsker's Theorem. By the law of large numbers, the first term in $H_{n}^{(2)}(t)$ converges in probability to $t E\left(\frac{\bar{X}_{i}^{(0)}}{\sqrt{1+\lambda \hat{\gamma}^{2}}}-c\right)^{2}=\frac{1+c^{2}\left(1+\lambda \hat{\gamma}^{2}\right)}{1+\lambda \hat{\gamma}^{2}} t$ for any $t$. It is also clear that the second term in $H_{n}^{(2)}(t)$ converges in probability to zero because $\bar{X}_{i}^{(j)}(\Delta t) \Delta t$ converges in probability to zero. 
The third term in $H_{n}^{(2)}(t)$ can be computed as follows:

$$
\begin{aligned}
& \sum_{i=1}^{[n t / T]}\left(\frac{\sum_{j=1}^{N_{i}(\Delta t)} \bar{X}_{i}^{(j)}(\Delta t) \sqrt{\Delta t}-\lambda \bar{\mu} \Delta t}{\sqrt{1+\lambda \hat{\gamma}^{2}}}\right)^{2} \\
= & \frac{1}{1+\lambda \hat{\gamma}^{2}}\left\{\sum_{i=1}^{[n t / T]}\left(\sum_{j=1}^{N_{i}(\Delta t)} \bar{X}_{i}^{(j)}(\Delta t) \sqrt{\Delta t}\right)^{2}-2 \lambda \bar{\mu} \sum_{i=1}^{[n t / T]} \sum_{j=1}^{N_{i}(\Delta t)} \bar{X}_{i}^{(j)}(\Delta t)(\Delta t)^{3 / 2}+\lambda^{2} \bar{\mu}^{2} \sum_{i=1}^{[n t / T]}(\Delta t)^{2}\right\} .
\end{aligned}
$$

The last two terms in the above expression clearly converge in probability to zero.

In order to deal with the remaining terms involving the Poisson random sum, we note that $\bar{X}_{i}^{(j)}(\Delta t) \sqrt{\Delta t}$ for different $i$ or $j$ are independent normal random variables with mean $\bar{\mu}$ and variance $\bar{\gamma}^{2}$. Thus,

$$
\sum_{j=1}^{N_{i}(\Delta t)} \bar{X}_{i}^{(j)}(\Delta t) \sqrt{\Delta t}=\bar{X}_{i}^{(1)}(\Delta t) \sqrt{\Delta t} 1_{\left\{N_{i}(\Delta t)=1\right\}}+o_{P}(\Delta t)
$$

and

$$
\left(\sum_{j=1}^{N_{i}(\Delta t)} \bar{X}_{i}^{(j)}(\Delta t) \sqrt{\Delta t}\right)^{2}=\left(\bar{X}_{i}^{(1)}(\Delta t) \sqrt{\Delta t}\right)^{2} 1_{\left\{N_{i}(\Delta t)=1\right\}}+o_{P}(\Delta t)
$$

where $o_{P}(\Delta t)$ denotes any term after dividing by $\Delta t$ converges to zero in probability. Note that $\lambda[n t / T] \Delta t$ converges to $\lambda t$. As a result, we have the following weak convergence:

$$
\left[\begin{array}{c}
\sum_{i=1}^{[n t / T]} \sum_{j=1}^{N_{i}(\Delta t)} \bar{X}_{i}^{(j)}(\Delta t) \sqrt{\Delta t} \\
\sum_{i=1}^{[n t / T]}\left(\sum_{j=1}^{N_{i}(\Delta t)} \bar{X}_{i}^{(j)}(\Delta t) \sqrt{\Delta t}\right)^{2}
\end{array}\right] \Longrightarrow\left[\begin{array}{c}
\int_{0}^{t}\left(\bar{\gamma} Z_{\pi_{s}}+\bar{\mu}\right) d \pi_{s} \\
\int_{0}^{t}\left(\bar{\gamma} Z_{\pi_{s}}+\bar{\mu}\right)^{2} d \pi_{s}
\end{array}\right] .
$$

Due to independence between the term that converges to the Brownian motion and those converge to the compound Poisson processes, we have

$$
\left[\begin{array}{l}
H_{n}^{(1)}(t) \\
H_{n}^{(2)}(t)
\end{array}\right] \Longrightarrow\left[\begin{array}{c}
W_{t}+\int_{0}^{t}\left(\bar{\gamma} Z_{\pi_{s}}+\bar{\mu}\right) d \pi_{s} \\
\frac{1+c^{2}\left(1+\lambda \hat{\gamma}^{2}\right)}{1+\lambda \hat{\gamma}^{2}} t+\frac{1}{1+\lambda \hat{\gamma}^{2}} \int_{0}^{t}\left(\bar{\gamma} Z_{\pi_{s}}+\bar{\mu}\right)^{2} d \pi_{s}
\end{array}\right] .
$$

The bivariate approximating system can be expressed as a system of stochastic differential equations with respect to $\left(H_{n}^{(1)}(t), H_{n}^{(2)}(t)\right)$. Applying Theorem 5.4 of Kurtz and Protter (1991) yields weak convergence to $\left(S_{t}, h_{t}\right)$. Thus, the limiting model under measure $\mathbf{P}$ is obtained.

\section{Proof of Proposition 2}

By substituting for $\bar{\mu}(\Delta t)$ and $\bar{\gamma}(\Delta t)$ in [Q1], under the Q measure, we obtain:

$$
\begin{aligned}
\ln S_{i \Delta t}-\ln S_{(i-1) \Delta t} & =\tilde{f}_{i \Delta t}(\Delta t)+\sqrt{h_{i \Delta t}} \tilde{J}_{i}(\Delta t) \sqrt{\Delta t} \\
\tilde{f}_{i \Delta t}(\Delta t) & =\left\{r-\frac{h_{i \Delta t}}{2}+\lambda \kappa\left[1-\exp \left(\sqrt{h_{i \Delta t}}(\bar{\mu}+b \rho \gamma \bar{\gamma})+\frac{1}{2} h_{i \Delta t} \bar{\gamma}^{2}\right)\right]\right\} \Delta t
\end{aligned}
$$

where $\tilde{J}_{i}(\Delta t)=\tilde{X}_{i}^{(0)}+\sum_{j=1}^{\tilde{N}_{i}(\Delta t)} \tilde{X}_{i}^{(j)}(\Delta t), \tilde{X}_{i}^{(0)} \sim N(0,1), \tilde{X}_{i}^{(j)}(\Delta t) \sim N\left(\frac{\bar{\mu}+b \rho \gamma \bar{\gamma}}{\sqrt{\Delta t}}, \frac{\bar{\gamma}^{2}}{\Delta t}\right), \tilde{N}_{i}(\Delta t)$ is a Poisson random variable with parameter $\lambda \kappa \Delta t$ and $\tilde{X}_{i}^{(j)}(j=0,1, \cdots)$ are independent. 
The approximate volatility dynamic can be deduced:

$$
\begin{aligned}
& h_{(i+1) \Delta t}-h_{i \Delta t} \\
= & \beta_{0} \Delta t+h_{i \Delta t}\left(\beta_{1}-1\right) \Delta t+\beta_{2} h_{i \Delta t}\left(\frac{\bar{J}_{i}(\Delta t)-\lambda \bar{\mu} \sqrt{\Delta t}}{\sqrt{1+\lambda \hat{\gamma}^{2}}}-c\right)^{2} \Delta t \\
= & \beta_{0} \Delta t+h_{i \Delta t}\left(\beta_{1}-1\right) \Delta t+\beta_{2}\left(\frac{1+\lambda \kappa \tilde{\gamma}^{2}}{1+\lambda \hat{\gamma}^{2}}\right) h_{i \Delta t}\left(\frac{\tilde{J}_{i}(\Delta t)-\lambda \kappa(\bar{\mu}+b \rho \gamma \bar{\gamma}) \sqrt{\Delta t}}{\sqrt{1+\lambda \kappa \tilde{\gamma}^{2}}}-c^{*}(\Delta t)\right)^{2} \Delta t
\end{aligned}
$$

where

$$
c^{*}(\Delta t)=c \sqrt{\frac{1+\lambda \hat{\gamma}^{2}}{1+\lambda \kappa \tilde{\gamma}^{2}}}+\frac{[\lambda \bar{\mu}-\lambda \kappa(\bar{\mu}+b \rho \gamma \bar{\gamma})-b \rho] \sqrt{\Delta t}}{\sqrt{1+\lambda \kappa \tilde{\gamma}^{2}}} .
$$

Note that the second equality in the volatility dynamic follows from $\bar{J}_{i}(\Delta t)=\tilde{J}_{i}(\Delta t)+b \rho \sqrt{\Delta t}$.

Similar to the case under measure $\mathrm{P}$, we define

$$
\begin{aligned}
\tilde{H}_{n}^{(1)}(t) & \equiv \sum_{i=1}^{[n t / T]} \tilde{J}_{i}(\Delta t) \sqrt{\Delta t} \\
\tilde{H}_{n}^{(2)}(t) & \equiv \sum_{i=1}^{[n t / T]}\left(\frac{\tilde{J}_{i}(\Delta t)-\lambda \kappa(\bar{\mu}+b \rho \gamma \bar{\gamma}) \sqrt{\Delta t}}{\sqrt{1+\lambda \kappa \tilde{\gamma}^{2}}}-c^{*}(\Delta t)\right)^{2} \Delta t .
\end{aligned}
$$

Applying the same arguments as in Proposition 1 yields

$$
\frac{\tilde{f}_{[n t / T] \Delta t}(\Delta t)}{\Delta t} \longrightarrow r-\frac{h_{t_{-}}}{2}+\lambda \kappa\left[1-\exp \left(\sqrt{h_{t_{-}}}(\bar{\mu}+b \rho \gamma \bar{\gamma})+\frac{1}{2} h_{t_{-}} \bar{\gamma}^{2}\right)\right]
$$

and

$$
\left[\begin{array}{c}
\tilde{H}_{n}^{(1)}(t) \\
\tilde{H}_{n}^{(2)}(t)
\end{array}\right] \Longrightarrow\left[\begin{array}{c}
\tilde{W}_{t}+\int_{0}^{t}\left(\bar{\gamma} \tilde{Z}_{\pi_{s}}+\bar{\mu}+b \rho \gamma \bar{\gamma}\right) d \tilde{\pi}_{s} \\
\frac{1+c^{2}\left(1+\lambda \hat{\gamma}^{2}\right)}{1+\lambda \kappa \tilde{\gamma}^{2}} t+\frac{1}{1+\lambda \kappa \tilde{\gamma}^{2}} \int_{0}^{t}\left(\bar{\gamma} \tilde{Z}_{\pi_{s}}+\bar{\mu}+b \rho \gamma \bar{\gamma}\right)^{2} d \tilde{\pi}_{s}
\end{array}\right] .
$$

By the same reason, the limiting model under measure $Q$ is obtained.

\section{Proof of Proposition 3}

First, we have the following result for the conditional mean return:

$$
\frac{f_{[n t / T] \Delta t}(\Delta t)}{\Delta t} \longrightarrow r-\frac{h_{t_{-}}}{2}-\sqrt{h_{t_{-}}} b \rho+\lambda \kappa\left[1-\exp \left(\sqrt{h_{t_{-}}}(\bar{\mu}+b \rho \gamma \bar{\gamma})+\frac{1}{2} h_{t_{-}} \bar{\gamma}^{2}\right)\right] .
$$

Next, define

$$
\begin{aligned}
H_{n}^{(1)}(t) & \equiv \sum_{i=1}^{[n t / T]} \bar{J}_{i}(\Delta t) \sqrt{\Delta t} \\
& =\sum_{i=1}^{[n t / T]} \bar{X}_{i}^{(0)} \sqrt{\Delta t}+\sum_{i=1}^{[n t / T]} \sum_{j=1}^{N_{i}(\Delta t)} \bar{X}_{i}^{(j)}(\Delta t) \sqrt{\Delta t} .
\end{aligned}
$$


Moreover,

$$
\begin{aligned}
H_{n}^{(2)}(t) & \equiv \sum_{i=1}^{[n t / T]}\left[\left|\frac{\bar{J}_{i}(\Delta t)-\lambda \bar{\mu} \sqrt{\Delta t}}{\sqrt{1+\lambda \hat{\gamma}^{2}}}\right|-q_{2}\right] \sqrt{\Delta t} \text { and } \\
H_{n}^{(3)}(t) & \equiv \sum_{i=1}^{[n t / T]}\left[\max \left(-\frac{\bar{J}_{i}(\Delta t)-\lambda \bar{\mu} \sqrt{\Delta t}}{\sqrt{1+\lambda \hat{\gamma}^{2}}}, 0\right)-q_{3}\right] \sqrt{\Delta t}
\end{aligned}
$$

Thus,

$$
\begin{aligned}
H_{n}^{(2)}(t)= & \sum_{i=1}^{[n t / T]} 1_{\left\{N_{i}(\Delta t)=0\right\}}\left[\left|\frac{\bar{X}_{i}^{(0)}-\lambda \bar{\mu} \sqrt{\Delta t}}{\sqrt{1+\lambda \hat{\gamma}^{2}}}\right|-q_{2}\right] \sqrt{\Delta t} \\
& +\sum_{i=1}^{[n t / T]} 1_{\left\{N_{i}(\Delta t) \neq 0\right\}}\left[\left|\frac{\bar{X}_{i}^{(0)} \sqrt{\Delta t}+\sum_{j=1}^{N_{i}(\Delta t)} \bar{X}_{i}^{(j)}(\Delta t) \sqrt{\Delta t}-\lambda \bar{\mu} \Delta t}{\sqrt{1+\lambda \hat{\gamma}^{2}}}\right|-q_{2} \sqrt{\Delta t}\right] \\
= & \sum_{i=1}^{[n t / T]}\left[\left|\frac{\bar{X}_{i}^{(0)}-\lambda \bar{\mu} \sqrt{\Delta t}}{\sqrt{1+\lambda \hat{\gamma}^{2}}}\right|-q_{2}\right] \sqrt{\Delta t} \\
& -\sum_{i=1}^{[n t / T]} 1_{\left\{N_{i}(\Delta t) \neq 0\right\}}\left[\left|\frac{\bar{X}_{i}^{(0)}-\lambda \bar{\mu} \sqrt{\Delta t}}{\sqrt{1+\lambda \hat{\gamma}^{2}}}\right|-q_{2}\right] \sqrt{\Delta t} \\
& +\sum_{i=1}^{[n t / T]} 1_{\left\{N_{i}(\Delta t) \neq 0\right\}}\left[\left|\frac{\bar{X}_{i}^{(0)} \sqrt{\Delta t}+\sum_{j=1}^{N_{i}(\Delta t)} \bar{X}_{i}^{(j)}(\Delta t) \sqrt{\Delta t}-\lambda \bar{\mu} \Delta t}{\sqrt{1+\lambda \hat{\gamma}^{2}}}\right|-q_{2} \sqrt{\Delta t}\right] \\
= & \sum_{i=1}^{[n t / T]}\left[\left|\frac{\bar{X}_{i}^{(0)}-\lambda \bar{\mu} \sqrt{\Delta t}}{\sqrt{1+\lambda \hat{\gamma}^{2}}}\right|-q_{2}\right] \sqrt{\Delta t}+\sum_{i=1}^{[n t / T]}\left|\frac{\sum_{j=1}^{N_{i}(\Delta t)} \bar{X}_{i}^{(j)}(\Delta t) \sqrt{\Delta t}}{\sqrt{1+\lambda \hat{\gamma}^{2}}}\right|+o_{P}(\sqrt{\Delta t}) .
\end{aligned}
$$

The last equality holds true because we collect the terms that are in the order of $\sqrt{\Delta t}$ or higher when a jump occurs, i.e., $1_{\left\{N_{i}(\Delta t) \neq 0\right\}}$ and denote it by $o_{P}(\sqrt{\Delta t})$. Similarly,

$$
\begin{aligned}
H_{n}^{(3)}(t)= & \sum_{i=1}^{[n t / T]}\left[\max \left(-\frac{\bar{X}_{i}^{(0)}-\lambda \bar{\mu} \sqrt{\Delta t}}{\sqrt{1+\lambda \hat{\gamma}^{2}}}, 0\right)-q_{3}\right] \sqrt{\Delta t} \\
& +\sum_{i=1}^{[n t / T]} \max \left(-\frac{\sum_{j=1}^{N_{i}(\Delta t)} \bar{X}_{i}^{(j)}(\Delta t) \sqrt{\Delta t}}{\sqrt{1+\lambda \hat{\gamma}^{2}}}, 0\right)+o_{P}(\sqrt{\Delta t}) .
\end{aligned}
$$

The first terms of $H_{n}^{(1)}(t), H_{n}^{(2)}(t)$ and $H_{n}^{(3)}(t)$ together have the following weak limit

$$
\left[\begin{array}{c}
\sum_{i=1}^{[n t / T]} \bar{X}_{i}^{(0)} \sqrt{\Delta t} \\
\sum_{i=1}^{[n t / T]}\left[\left|\frac{\bar{X}_{i}^{(0)}-\lambda \bar{\mu} \sqrt{\Delta t}}{\sqrt{1+\lambda \hat{\gamma}^{2}}}\right|-q_{2}\right] \sqrt{\Delta t} \\
\sum_{i=1}^{[n t / T]}\left[\max \left(-\frac{\bar{X}_{i}^{(0)}-\lambda \bar{\mu} \sqrt{\Delta t}}{\sqrt{1+\lambda \hat{\gamma}^{2}}}, 0\right)-q_{3}\right] \sqrt{\Delta t}
\end{array}\right] \Longrightarrow\left[\begin{array}{c}
W_{t} \\
\sqrt{\frac{\pi-2}{\pi\left(1+\lambda \hat{\gamma}^{2}\right)}} B_{t} \\
\frac{\lambda \bar{\mu}}{2 \sqrt{\left.1+\lambda \hat{\gamma}^{2}\right)}} t-\frac{1}{2 \sqrt{1+\lambda \hat{\gamma}^{2}}} W_{t}+\sqrt{\frac{\pi-2}{4 \pi\left(1+\lambda \hat{\gamma}^{2}\right)}} B_{t}
\end{array}\right] .
$$

The weak convergence of the first element is standard. For the second and third elements, we can still apply the standard weak convergence result as long as we properly deal with the extra term, $\lambda \bar{\mu} \sqrt{\Delta t}$. First note that

$$
\frac{1}{\sqrt{\Delta t}}\left[E\left|\frac{\bar{X}_{i}^{(0)}-\lambda \bar{\mu} \sqrt{\Delta t}}{\sqrt{1+\lambda \hat{\gamma}^{2}}}\right|-q_{2}\right] \rightarrow 0
$$


and

$$
\operatorname{Var}\left[\left|\frac{\bar{X}_{i}^{(0)}-\lambda \bar{\mu} \sqrt{\Delta t}}{\sqrt{1+\lambda \hat{\gamma}^{2}}}\right|\right] \rightarrow \frac{\pi-2}{\pi\left(1+\lambda \hat{\gamma}^{2}\right)} .
$$

Thus, one can effectively ignore $\lambda \bar{\mu} \sqrt{\Delta t}$ as far as the limit for the second element is concerned. Next we note that

$$
\frac{1}{\sqrt{\Delta t}}\left[E \max \left(-\frac{\bar{X}_{i}^{(0)}-\lambda \bar{\mu} \sqrt{\Delta t}}{\sqrt{1+\lambda \hat{\gamma}^{2}}}, 0\right)-q_{3}\right] \rightarrow \frac{\lambda \bar{\mu}}{2 \sqrt{1+\lambda \hat{\gamma}^{2}}}
$$

and

$$
\operatorname{Var}\left[\max \left(-\frac{\bar{X}_{i}^{(0)}-\lambda \bar{\mu} \sqrt{\Delta t}}{\sqrt{1+\lambda \hat{\gamma}^{2}}}, 0\right)\right] \rightarrow \frac{\pi-1}{2 \pi\left(1+\lambda \hat{\gamma}^{2}\right)} .
$$

Thus, the third element must contain an extra term $\frac{\lambda \bar{\mu}}{2 \sqrt{1+\lambda \hat{\gamma}^{2}}} t$.

We now turn to the terms involving the Poisson random sum, we note that $\bar{X}_{i}^{(j)}(\Delta t) \sqrt{\Delta t}$ for different $i$ or $j$ are independent normal random variables with mean $\bar{\mu}$ and variance $\bar{\gamma}^{2}$. Thus,

$$
\sum_{j=1}^{N_{i}(\Delta t)} \bar{X}_{i}^{(j)}(\Delta t) \sqrt{\Delta t}=\bar{X}_{i}^{(1)}(\Delta t) \sqrt{\Delta t} 1_{\left\{N_{i}(\Delta t)=1\right\}}+o_{P}(\Delta t)
$$

and

$$
\left|\sum_{j=1}^{N_{i}(\Delta t)} \bar{X}_{i}^{(j)}(\Delta t) \sqrt{\Delta t}\right|=\left|\bar{X}_{i}^{(1)}(\Delta t) \sqrt{\Delta t}\right| 1_{\left\{N_{i}(\Delta t)=1\right\}}+o_{P}(\Delta t) .
$$

As a result, we have the following weak convergence:

$$
\left[\begin{array}{c}
\sum_{i=1}^{[n t / T]} \sum_{j=1}^{N_{i}(\Delta t) \bar{X}_{i}^{(j)}(\Delta t) \sqrt{\Delta t}} \\
\sum_{i=1}^{[n t / T]}\left|\frac{\sum_{j=1}^{N_{i}(\Delta t)} \bar{X}_{i}^{(j)}(\Delta t) \sqrt{\Delta t}}{\sqrt{1+\lambda \hat{\gamma}^{2}}}\right| \\
\sum_{i=1}^{[n t / T]} \max \left(-\frac{\sum_{j=1}^{N_{i}(\Delta t)} \bar{X}_{i}^{(j)}(\Delta t) \sqrt{\Delta t}}{\sqrt{1+\lambda \hat{\gamma}^{2}}}, 0\right)
\end{array}\right] \Longrightarrow\left[\begin{array}{c}
\int_{0}^{t}\left(\bar{\gamma} Z_{\pi_{s}}+\bar{\mu}\right) d \pi_{s} \\
\frac{1}{\sqrt{1+\lambda \hat{\gamma}^{2}}} \int_{0}^{t}\left|\bar{\gamma} Z_{\pi_{s}}+\bar{\mu}\right| d \pi_{s} \\
\frac{1}{\sqrt{1+\lambda \hat{\gamma}^{2}}} \int_{0}^{t} \max \left(-\bar{\gamma} Z_{\pi_{s}}-\bar{\mu}, 0\right) d \pi_{s}
\end{array}\right] .
$$

Due to independence between the terms that converge to the Brownian motions and those converge to the compound Poisson processes, we have

$$
\left[\begin{array}{c}
H_{n}^{(1)}(t) \\
H_{n}^{(2)}(t) \\
H_{n}^{(3)}(t)
\end{array}\right] \Longrightarrow\left[\begin{array}{c}
W_{t}+\int_{0}^{t}\left(\bar{\gamma} Z_{\pi_{s}}+\bar{\mu}\right) d \pi_{s} \\
\sqrt{\frac{\pi-2}{\pi\left(1+\lambda \hat{\gamma}^{2}\right)}} B_{t}+\frac{1}{\sqrt{1+\lambda \hat{\gamma}^{2}}} \int_{0}^{t}\left|\bar{\gamma} Z_{\pi_{s}}+\bar{\mu}\right| d \pi_{s} \\
\frac{\lambda \bar{\mu}}{2 \sqrt{\left.1+\lambda \hat{\gamma}^{2}\right)}} t-\frac{1}{2 \sqrt{1+\lambda \hat{\gamma}^{2}}} W_{t}+\sqrt{\frac{\pi-2}{4 \pi\left(1+\lambda \hat{\gamma}^{2}\right)}} B_{t}+\frac{1}{\sqrt{1+\lambda \hat{\gamma}^{2}}} \int_{0}^{t} \max \left(-\bar{\gamma} Z_{\pi_{s}}-\bar{\mu}, 0\right) d \pi_{s}
\end{array}\right] .
$$

The bivariate approximating system can be expressed as a system of stochastic differential equations with respect to $\left(H_{n}^{(1)}(t), H_{n}^{(2)}(t), H_{n}^{(3)}(t)\right)$. Applying Theorem 5.4 of Kurtz and Protter (1991) yields weak convergence to $\left(S_{t}, \phi_{t}\right)$. Thus, the limiting model under measure $\mathrm{P}$ is obtained.

\section{Proof of Proposition 4}

We observe that the approximating bivariate system under measure $\mathrm{Q}$ is the same as that under measure $\mathrm{P}$ except that (i) the volatility dynamic under measure Q contains $(b \rho-\lambda \bar{\mu}) \sqrt{\Delta t}$ as opposed to $-\lambda \bar{\mu} \sqrt{\Delta t}$, (ii) 
$\tilde{X}_{i}^{(j)}(\Delta t) \sim N\left(\frac{\bar{\mu}+b \rho \gamma \bar{\gamma}}{\sqrt{\Delta t}}, \frac{\bar{\gamma}^{2}}{\Delta t}\right)$ under measure Q as opposed to $\bar{X}_{i}^{(j)}(\Delta t) \sim N\left(\frac{\bar{\mu}}{\sqrt{\Delta t}}, \frac{\bar{\gamma}^{2}}{\Delta t}\right)$ under measure P, and (iii) $\tilde{N}_{i}(\Delta t)$ has $\lambda \kappa$ as the parameter as opposed to $N_{i}(\Delta t)$ is governed by parameter $\lambda$. The statement of this proposition can thus be established in the same way as that under measure $\mathrm{P}$.

\section{Proof of Proposition 5}

The model under measure $\mathbf{P}$ is directly obtained from [P1] given the assumed structure for $\bar{\mu}(\Delta t)$ and $\bar{\gamma}(\Delta t)$. We have the following result for the conditional mean:

$$
\frac{f_{[n t / T] \Delta t}(\Delta t)}{\Delta t} \longrightarrow r-\frac{h_{t_{-}}}{2}-\sqrt{h_{t_{-}}} b \rho .
$$

Next, define

$$
\begin{aligned}
H_{n}^{(1)}(t) & \equiv \sum_{i=1}^{[n t / T]} \bar{J}_{i}(\Delta t) \sqrt{\Delta t} \\
& =\sum_{i=1}^{[n t / T]} \bar{X}_{i}^{(0)} \sqrt{\Delta t}+\sum_{i=1}^{[n t / T]} \sum_{j=1}^{N_{i}(\Delta t)} \bar{X}_{i}^{(j)}(\Delta t) \sqrt{\Delta t}
\end{aligned}
$$

The second term in the above equation converges to zero in probability because $\bar{X}_{i}^{(j)}(\Delta t) \sqrt{\Delta t}$ converges to zero in probability. Moreover,

$$
\begin{aligned}
H_{n}^{(2)}(t) \equiv & \sum_{i=1}^{[n t / T]}\left[\left(\frac{\bar{J}_{i}(\Delta t)-\lambda \bar{\mu}(\Delta t)^{3 / 4}}{\sqrt{1+\lambda \hat{\gamma}^{2} \sqrt{\Delta t}}}-c\right)^{2}-\left(1+c^{2}\right)\right] \sqrt{\Delta t} \\
= & \left.\sum_{i=1}^{[n t / T]}\left[\left(\frac{\bar{X}_{i}^{(0)}}{\sqrt{1+\lambda \hat{\gamma}^{2} \sqrt{\Delta t}}}-c\right)^{2}-\left(1+c^{2}\right)\right] \sqrt{\Delta t}\right] \\
& +2 \sum_{i=1}^{[n t / T]}\left(\frac{\bar{X}_{i}^{(0)}}{\sqrt{1+\lambda \hat{\gamma}^{2} \sqrt{\Delta t}}}-c\right)\left(\frac{\sum_{j=1}^{N_{i}(\Delta t)} \bar{X}_{i}^{(j)}(\Delta t) \sqrt{\Delta t}-\lambda \bar{\mu}(\Delta t)^{5 / 4}}{\sqrt{1+\lambda \hat{\gamma}^{2} \sqrt{\Delta t}}}\right) \\
& +\sum_{i=1}^{[n t / T]}\left(\frac{\sum_{j=1}^{N_{i}(\Delta t)} \bar{X}_{i}^{(j)}(\Delta t)(\Delta t)^{1 / 4}-\lambda \bar{\mu} \Delta t}{\sqrt{1+\lambda \hat{\gamma}^{2} \sqrt{\Delta t}}}\right)^{2} .
\end{aligned}
$$

Note that the second term in the above equation also converges to zero in probability because $\bar{X}_{i}^{(j)}(\Delta t) \sqrt{\Delta t}$ converges to zero in probability.

Then note that

$$
\begin{aligned}
& \sum_{i=1}^{[n t / T]}\left[\left(\frac{\bar{X}_{i}^{(0)}}{\sqrt{1+\lambda \hat{\gamma}^{2} \sqrt{\Delta t}}}-c\right)^{2}-\left(1+c^{2}\right)\right] \sqrt{\Delta t} \\
= & \frac{1}{1+\lambda \hat{\gamma}^{2} \sqrt{\Delta t}} \sum_{i=1}^{[n t / T]}\left[\left(\bar{X}_{i}^{(0)}-c \sqrt{1+\lambda \hat{\gamma}^{2} \sqrt{\Delta t}}\right)^{2}-\left(1+c^{2}\right)\left(1+\lambda \hat{\gamma}^{2} \sqrt{\Delta t}\right)\right] \sqrt{\Delta t}
\end{aligned}
$$




$$
\begin{aligned}
= & \frac{1}{1+\lambda \hat{\gamma}^{2} \sqrt{\Delta t}} \sum_{i=1}^{[n t / T]}\left[\left(\bar{X}_{i}^{(0)}-c \sqrt{1+\lambda \hat{\gamma}^{2} \sqrt{\Delta t}}\right)^{2}-\left[1+c^{2}\left(1+\lambda \hat{\gamma}^{2} \sqrt{\Delta t}\right)\right]\right] \sqrt{\Delta t} \\
& -\frac{\lambda \hat{\gamma}^{2}}{1+\lambda \hat{\gamma}^{2} \sqrt{\Delta t}} \sum_{i=1}^{[n t / T]} \Delta t .
\end{aligned}
$$

The standard weak convergence argument leads to:

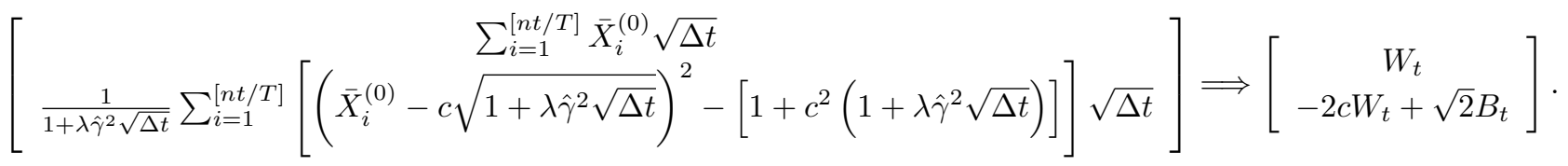

In addition, we have

$$
\frac{\lambda \hat{\gamma}^{2}}{1+\lambda \hat{\gamma}^{2} \sqrt{\Delta t}} \sum_{i=1}^{[n t / T]} \Delta t \longrightarrow \lambda \hat{\gamma}^{2} t
$$

For the term involving the Poisson random sum in $H_{n}^{(2)}(t)$, we follow the same argument as in the proof for Proposition 1 to obtain:

$$
\sum_{i=1}^{[n t / T]}\left(\frac{\sum_{j=1}^{N_{i}(\Delta t)} \bar{X}_{i}^{(j)}(\Delta t)(\Delta t)^{1 / 4}-\lambda \bar{\mu} \Delta t}{\sqrt{1+\lambda \hat{\gamma}^{2} \sqrt{\Delta t}}}\right)^{2} \Longrightarrow \int_{0}^{t}\left(\bar{\gamma} Z_{\pi_{s}}+\bar{\mu}\right)^{2} d \pi_{s}
$$

because $\bar{X}_{i}^{(1)}(\Delta t)(\Delta t)^{1 / 4}$ is a normal random variable with mean $\bar{\mu}$ and variance $\bar{\gamma}^{2}$. Again due to independence between the Brownian motions and the compound Poisson process, we have

$$
\left[\begin{array}{c}
H_{n}^{(1)}(t) \\
H_{n}^{(2)}(t)
\end{array}\right] \Longrightarrow\left[\begin{array}{c}
W_{t} \\
-\lambda \hat{\gamma}^{2} t-2 c W_{t}+\sqrt{2} B_{t}+\int_{0}^{t}\left(\bar{\gamma} Z_{\pi_{s}}+\bar{\mu}\right)^{2} d \pi_{s}
\end{array}\right]
$$

Applying Theorem 5.4 of Kurtz and Protter (1991) yields the weak convergence to the limiting model under measure $\mathrm{P}$.

\section{Proof of Proposition 6}

Under the assumed structure for $\bar{\mu}(\cdot)$ and $\bar{\gamma}(\cdot)$, we have:

$$
\begin{aligned}
E^{\mathrm{Q}}\left(\tilde{J}_{i}(\Delta t)\right) & =\tilde{\lambda}(\Delta t)\left(\bar{\mu}(\Delta t)^{-1 / 4}+b \rho \gamma \bar{\gamma}\right) \\
\left.\operatorname{Var}_{(} \tilde{J}_{i}(\Delta t)\right) & =1+\tilde{\lambda}(\Delta t) \tilde{\gamma}^{2}(\Delta t)
\end{aligned}
$$

where

$$
\begin{aligned}
\tilde{\gamma}^{2}(\Delta t) & =\left(\bar{\mu}(\Delta t)^{-1 / 4}+b \rho \gamma \bar{\gamma}\right)^{2}+\frac{\bar{\gamma}^{2}}{\sqrt{\Delta t}} \\
\tilde{\lambda}(\Delta t) & =\lambda \kappa(\Delta t) \Delta t \\
\kappa(\Delta t) & =e^{b \mu(\Delta t)^{1 / 4}+\frac{1}{2} b^{2} \gamma^{2} \sqrt{\Delta t}} .
\end{aligned}
$$

The volatility scaling updating scheme given in equation (40) can be reexpressed as

$$
\begin{aligned}
h_{(i+1) \Delta t}-h_{i \Delta t}= & \beta_{0} \Delta t+h_{i \Delta t}\left(\beta_{1}+\beta_{2}\left(1+c^{2}\right)-1\right) \Delta t+ \\
& \beta_{2}^{*}(\Delta t) h_{i \Delta t}\left[\left(\frac{\tilde{J}_{i}(\Delta t)-E^{\mathrm{Q}}\left(\tilde{J}_{i}(\Delta t)\right)}{\left.\left.\sqrt{\operatorname{Var} \mathrm{Q}\left(\tilde{J}_{i}(\Delta t)\right)}-c^{*}(\Delta t)\right)^{2}-d^{*}(\Delta t)\right] \sqrt{\Delta t}}\right.\right.
\end{aligned}
$$


where

$$
\begin{aligned}
c^{*}(\Delta t) & =\frac{E^{\mathrm{P}}\left(\tilde{J}_{i}(\Delta t)\right)-E^{\mathrm{Q}}\left(\tilde{J}_{i}(\Delta t)\right)-b \rho \sqrt{\Delta t}+c \sqrt{\operatorname{Var}^{\mathrm{P}}\left(\tilde{J}_{i}(\Delta t)\right)}}{\sqrt{\operatorname{Var} \mathrm{Q}\left(\tilde{J}_{i}(\Delta t)\right)}} \\
\beta_{2}^{*}(\Delta t) & =\beta_{2} \frac{\operatorname{Var}^{\mathrm{Q}}\left(\tilde{J}_{i}(\Delta t)\right)}{\operatorname{Var}^{\mathrm{P}\left(\tilde{J}_{i}(\Delta t)\right)}} \\
d^{*}(\Delta t) & =\left(1+c^{2}\right) \frac{\operatorname{Var}^{\mathrm{P}}\left(\tilde{J}_{i}(\Delta t)\right)}{\operatorname{Var}^{\mathrm{Q}}\left(\tilde{J}_{i}(\Delta t)\right)} .
\end{aligned}
$$

Now, note that

$$
\begin{aligned}
\frac{\tilde{\lambda}(\Delta t)}{\Delta t} & =\lambda \exp \left(b \mu(\Delta t)^{1 / 4}+\frac{1}{2} b^{2} \gamma^{2} \sqrt{\Delta t}\right) \longrightarrow \lambda \\
\tilde{\lambda}(\Delta t) \tilde{\gamma}^{2}(\Delta t) & =\lambda \Delta t \exp \left(b \mu(\Delta t)^{1 / 4}+\frac{1}{2} b^{2} \gamma^{2} \sqrt{\Delta t}\right)\left[\left(\frac{\bar{\mu}}{(\Delta t)^{1 / 4}}+b \rho \gamma \bar{\gamma}\right)^{2}+\frac{\bar{\gamma}^{2}}{\sqrt{\Delta t}}\right] \longrightarrow 0 \\
\beta_{2}^{*}(\Delta t) & =\beta_{2}\left(\frac{1+\tilde{\lambda}(\Delta t) \tilde{\gamma}^{2}(\Delta t)}{1+\lambda \hat{\gamma}^{2} \sqrt{\Delta t}}\right) \longrightarrow \beta_{2} \\
c^{*}(\Delta t) & =\frac{c \sqrt{1+\lambda \hat{\gamma}^{2} \sqrt{\Delta t}}+\lambda \bar{\mu}(\Delta t)^{3 / 4}-\tilde{\lambda}(\Delta t)\left(\frac{\bar{\mu}}{(\Delta t)^{1 / 4}}+b \rho \gamma \bar{\gamma}\right)-b \rho \sqrt{\Delta t}}{\sqrt{1+\tilde{\lambda}(\Delta t) \tilde{\gamma}^{2}(\Delta t)}} \longrightarrow c .
\end{aligned}
$$

Moreover,

$$
\begin{aligned}
& \frac{1+\left(c^{* 2}(\Delta t)-d^{*}(\Delta t)\right)\left(1+\tilde{\lambda}(\Delta t) \tilde{\gamma}^{2}(\Delta t)\right)}{\sqrt{\Delta t}} \\
= & \frac{1}{\sqrt{\Delta t}}\left\{1+c^{2}\left(1+\lambda \hat{\gamma}^{2} \sqrt{\Delta t}\right)-2 c b \rho \sqrt{\Delta t}+o(\sqrt{\Delta t})-\left(1+c^{2}\right)\left(1+\lambda \hat{\gamma}^{2} \sqrt{\Delta t}\right)\right\} \\
= & \frac{1}{\sqrt{\Delta t}}\left\{-2 \operatorname{cb} \rho \sqrt{\Delta t}-\lambda \hat{\gamma}^{2} \sqrt{\Delta t}+o(\sqrt{\Delta t})\right\} \\
\longrightarrow & -2 c b \rho-\lambda \hat{\gamma}^{2}
\end{aligned}
$$

The conditional mean process has the following limit:

$$
\frac{\tilde{f}_{[n t / T] \Delta t}(\Delta t)}{\Delta t} \longrightarrow r-\frac{h_{t_{-}}}{2} .
$$

Similar to the case under measure $\mathrm{P}$,

$$
\begin{aligned}
\tilde{H}_{n}^{(1)}(t) & \equiv \sum_{i=1}^{[n t / T]} \tilde{J}_{i}(\Delta t) \sqrt{\Delta t} \\
& =\sum_{i=1}^{[n t / T]} \tilde{X}_{i}^{(0)} \sqrt{\Delta t}+\sum_{i=1}^{[n t / T]} \sum_{j=1}^{\tilde{N}_{i}(\Delta t)} \tilde{X}_{i}^{(j)}(\Delta t) \sqrt{\Delta t} .
\end{aligned}
$$

The second term in the above equation converges to zero in probability because $\tilde{X}_{i}^{(j)}(\Delta t) \sqrt{\Delta t}$ converges to zero in probability. Moreover,

$$
\tilde{H}_{n}^{(2)}(t)
$$




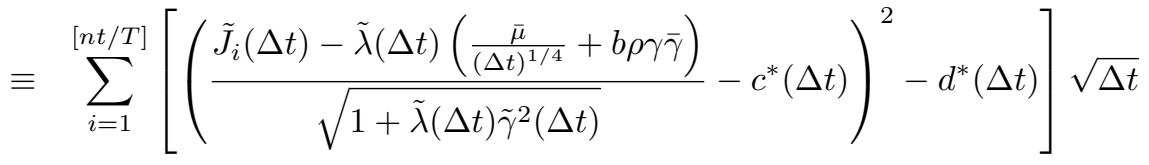

$$
\begin{aligned}
& =\sum_{i=1}^{[n t / T]}\left[\left(\frac{\tilde{X}_{i}^{(0)}}{\sqrt{1+\tilde{\lambda}(\Delta t) \tilde{\gamma}^{2}(\Delta t)}}-c^{*}(\Delta t)\right)^{2}-d^{*}(\Delta t)\right] \sqrt{\Delta t} \\
& +2 \sum_{i=1}^{[n t / T]}\left(\frac{\tilde{X}_{i}^{(0)}}{\sqrt{1+\tilde{\lambda}(\Delta t) \tilde{\gamma}^{2}(\Delta t)}}-c^{*}(\Delta t)\right)\left(\frac{\sum_{j=1}^{\tilde{N}_{i}(\Delta t)} \tilde{X}_{i}^{(j)}(\Delta t) \sqrt{\Delta t}-\tilde{\lambda}(\Delta t)\left(\frac{\bar{\mu}}{(\Delta t)^{1 / 4}}+b \rho \gamma \bar{\gamma}\right) \sqrt{\Delta t}}{\sqrt{1+\tilde{\lambda}(\Delta t) \tilde{\gamma}^{2}(\Delta t)}}\right) \\
& +\sum_{i=1}^{[n t / T]}\left(\frac{\sum_{j=1}^{\tilde{N}_{i}(\Delta t)} \tilde{X}_{i}^{(j)}(\Delta t)(\Delta t)^{1 / 4}-\tilde{\lambda}(\Delta t)\left(\frac{\bar{\mu}}{(\Delta t)^{1 / 4}}+b \rho \gamma \bar{\gamma}\right)(\Delta t)^{1 / 4}}{\sqrt{1+\tilde{\lambda}(\Delta t) \tilde{\gamma}^{2}(\Delta t)}}\right)^{2} .
\end{aligned}
$$

Note that the second term in the above equation also converges to zero in probability because $\tilde{X}_{i}^{(j)}(\Delta t) \sqrt{\Delta t}$ and $\tilde{\lambda}(\Delta t) \sqrt{\Delta t}$ converges to zero.

The weak convergence argument similar to Proposition 5 leads to:

$$
\left[\sum_{i=1}^{[n t / T]}\left[\left(\frac{\sum_{i=1}^{[n t / T]} \tilde{X}_{i}^{(0)} \sqrt{\Delta t}}{\sqrt{1+\tilde{\lambda}(\Delta t) \tilde{\gamma}^{2}(\Delta t)}}-c^{*}(\Delta t)\right)^{2}-d^{*}(\Delta t)\right] \sqrt{\Delta t}\right] \Longrightarrow\left[\begin{array}{c}
\tilde{W}_{t} \\
-\left(2 c b \rho+\lambda \hat{\gamma}^{2}\right) t-2 c \tilde{W}_{t}+\sqrt{2} \tilde{B}_{t}
\end{array}\right] .
$$

The extra term $-\left(2 c b \rho+\lambda \hat{\gamma}^{2}\right) t$ is due to the fact that $\frac{1+\left(c^{* 2}(\Delta t)-d^{*}(\Delta t)\right)\left(1+\tilde{\lambda}(\Delta t) \tilde{\gamma}^{2}(\Delta t)\right)}{\sqrt{\Delta t}} \longrightarrow-2 c b \rho-\lambda \hat{\gamma}^{2}$, a result established earlier. For the term involving the Poisson random sum in $\tilde{H}_{n}^{(2)}(t)$, we have

$$
\sum_{i=1}^{[n t / T]}\left(\frac{\sum_{j=1}^{\tilde{N}_{i}(\Delta t)} \tilde{X}_{i}^{(j)}(\Delta t)(\Delta t)^{1 / 4}-\tilde{\lambda}(\Delta t)\left(\frac{\bar{\mu}}{(\Delta t)^{1 / 4}}+b \rho \gamma \bar{\gamma}\right)(\Delta t)^{1 / 4}}{\sqrt{1+\tilde{\lambda}(\Delta t) \tilde{\gamma}^{2}(\Delta t)}}\right)^{2} \Longrightarrow \int_{0}^{t}\left(\bar{\gamma} \tilde{Z}_{\pi_{s}}+\bar{\mu}\right)^{2} d \tilde{\pi}_{s}
$$

because $\tilde{X}_{i}^{(1)}(\Delta t)(\Delta t)^{1 / 4}$ is a normal random variable with mean $\bar{\mu}$ and variance $\bar{\gamma}^{2}$. Again due to independence between the Brownian motions and the compound Poisson process, we have

$$
\left[\begin{array}{c}
\tilde{H}_{n}^{(1)}(t) \\
\tilde{H}_{n}^{(2)}(t)
\end{array}\right] \Longrightarrow\left[\begin{array}{c}
\tilde{W}_{t} \\
-\left(2 c b \rho+\lambda \hat{\gamma}^{2}\right) t-2 c \tilde{W}_{t}+\sqrt{2} \tilde{B}_{t}+\int_{0}^{t}\left(\bar{\gamma}_{\pi_{s}}+\bar{\mu}\right)^{2} d \tilde{\pi}_{s}
\end{array}\right]
$$

Thus, the limiting model under measure $\mathrm{Q}$ is obtained. 
Table 1

Convergence of Percentage Errors in Option Prices for Case 1

The Table shows the percentage errors in option prices using the Euler and approximating GARCH-Jump schemes. The top entries show the convergence behavior of errors when the Euler approximation scheme is used. The bottom entries in each row show the errors when the approximating GARCH-Jump option model is used. The models are shown below where the relevant variables are defined in Proposition 2. The percentage error is defined as the GARCH (or Euler) price minus the true price divided by the true price, and reported as a percentage. The true price is the Euler price based on 1024 partitions per day. The parameter values used are discussed in the text. The option contracts are European calls with 30 days to expiration. In all cases 50000 simulations were used using antithetic control variables The specific approximating GARCH-Jump model, corresponding to [Q1] for Case 1 reduces to:

$$
\begin{aligned}
S_{i \Delta t} & =S_{(i-1) \Delta t} e^{\tilde{f}_{i \Delta t}(\Delta t)+\sqrt{h_{i \Delta t}} \tilde{J}_{i}(\Delta t) \sqrt{\Delta t}} \\
h_{(i+1) \Delta t}-h_{i \Delta t} & =\beta_{0} \Delta t+h_{i \Delta t}\left(\beta_{1}-1\right) \Delta t \\
& +\beta_{2}\left(\frac{1+\lambda \kappa \tilde{\gamma}^{2}}{1+\lambda \hat{\gamma}^{2}}\right) h_{i \Delta t}\left(\frac{\tilde{J}_{i}(\Delta t)-\lambda \kappa(\bar{\mu}+b \rho \gamma \bar{\gamma}) \sqrt{\Delta t}}{\sqrt{1+\lambda \kappa \tilde{\gamma}^{2}}}-c^{*}(\Delta t)\right)^{2} \Delta t
\end{aligned}
$$

where $\tilde{J}_{i}(\Delta t)=\tilde{X}_{i}^{(0)}+\Sigma_{j=1}^{\tilde{N}_{i}(\Delta t)} \tilde{X}_{i}^{(j)}(\Delta t)$, with $\tilde{X}_{i}^{(0)} \sim N(0,1)$ and $\tilde{X}_{i}^{(j)}(\Delta t) \sim N\left(\frac{\bar{\mu}+b \rho \gamma \bar{\gamma}}{\sqrt{\Delta t}}, \frac{\bar{\gamma}^{2}}{\Delta t}\right)$ and $\tilde{N}_{i}(\Delta t)$ is a Poisson random variable with parameter $\lambda \kappa \Delta t$ and

$$
\begin{aligned}
\tilde{f}_{i \Delta t}(\Delta t) & =\left(r-\frac{h_{i \Delta t}}{2}\right) \Delta t+\lambda \kappa\left(1-K_{i}\left(h_{i \Delta t}\right)\right) \Delta t \\
K_{i}\left(h_{i \Delta t}\right) & =e^{\sqrt{h_{i \Delta t}}(\bar{\mu}+b \rho \gamma \bar{\gamma})+\frac{1}{2} h_{i \Delta t} \bar{\gamma}^{2}} \\
c^{*}(\Delta t) & =c \sqrt{\frac{1+\lambda \hat{\gamma}^{2}}{1+\lambda \kappa \tilde{\gamma}^{2}}}+\frac{[\lambda \bar{\mu}-\lambda \kappa(\bar{\mu}+b \rho \gamma \bar{\gamma})-b \rho] \sqrt{\Delta t}}{\sqrt{1+\lambda \kappa \tilde{\gamma}^{2}}}
\end{aligned}
$$

\begin{tabular}{|c|c|c|c|c|c|c|c|c|c|c|c|}
\hline \multicolumn{12}{|c|}{ Number of Partitions per Day } \\
\hline Strike & 1 & 2 & 4 & 8 & 16 & 32 & 64 & 128 & 256 & 512 & 1024 \\
\hline \multirow{2}{*}{450} & 61.36 & 32.89 & 17.36 & 9.41 & 4.47 & 2.44 & 1.08 & 0.23 & 0.48 & 0.66 & - \\
\hline & 3.02 & 2.63 & 1.87 & 1.78 & 1.05 & 0.82 & 0.36 & -0.12 & 0.34 & 0.58 & -0.061 \\
\hline \multirow{2}{*}{460} & 68.03 & 36.60 & 19.37 & 10.52 & 5.03 & 2.75 & 1.25 & 0.29 & 0.56 & 0.76 & - \\
\hline & 3.47 & 3.05 & 2.19 & 2.08 & 1.25 & 0.98 & 0.50 & -0.06 & 0.44 & 0.70 & -0.032 \\
\hline \multirow{2}{*}{470} & 75.41 & 40.67 & 21.56 & 11.75 & 5.63 & 3.08 & 1.43 & 0.34 & 0.65 & 0.86 & - \\
\hline & 3.99 & 3.51 & 2.55 & 2.43 & 1.48 & 1.17 & 0.64 & 0.01 & 0.56 & 0.84 & 0.002 \\
\hline \multirow{2}{*}{480} & 83.55 & 45.14 & 23.95 & 13.07 & 6.29 & 3.44 & 1.61 & 0.40 & 0.74 & 0.98 & - \\
\hline & 4.56 & 4.03 & 2.95 & 2.82 & 1.74 & 1.38 & 0.79 & 0.08 & 0.69 & 1.00 & 0.044 \\
\hline \multirow{2}{*}{490} & 92.52 & 50.01 & 26.55 & 14.48 & 6.97 & 3.82 & 1.78 & 0.44 & 0.82 & 1.09 & - \\
\hline & 5.21 & 4.59 & 3.39 & 3.22 & 2.02 & 1.60 & 0.93 & 0.15 & 0.81 & 1.16 & 0.096 \\
\hline \multirow{2}{*}{500} & 102.40 & 55.32 & 29.35 & 15.99 & 7.69 & 4.21 & 1.96 & 0.49 & 0.90 & 1.21 & - \\
\hline & 5.94 & 5.22 & 3.87 & 3.65 & 2.31 & 1.84 & 1.08 & 0.22 & 0.95 & 1.35 & 0.158 \\
\hline \multirow{2}{*}{510} & 113.29 & 61.08 & 32.38 & 17.60 & 8.46 & 4.64 & 2.13 & 0.54 & 0.99 & 1.33 & - \\
\hline & 6.77 & 5.91 & 4.40 & 4.10 & 2.62 & 2.12 & 1.24 & 0.31 & 1.11 & 1.54 & 0.234 \\
\hline \multirow{2}{*}{520} & 125.23 & 67.34 & 35.63 & 19.32 & 9.24 & 5.09 & 2.31 & 0.59 & 1.08 & 1.45 & - \\
\hline & 7.66 & 6.66 & 4.97 & 4.56 & 2.93 & 2.42 & 1.41 & 0.40 & 1.28 & 1.75 & 0.323 \\
\hline \multirow{2}{*}{530} & 138.34 & 74.13 & 39.14 & 21.14 & 10.06 & 5.56 & 2.49 & 0.63 & 1.15 & 1.59 & - \\
\hline & 8.66 & 7.51 & 5.58 & 5.07 & 3.28 & 2.74 & 1.58 & 0.51 & 1.45 & 1.98 & 0.423 \\
\hline \multirow{2}{*}{540} & 152.70 & 81.48 & 42.90 & 23.08 & 10.94 & 6.03 & 2.63 & 0.66 & 1.22 & 1.72 & - \\
\hline & 9.79 & 8.44 & 6.25 & 5.63 & 3.65 & 3.07 & 1.75 & 0.61 & 1.62 & 2.22 & 0.529 \\
\hline \multirow{2}{*}{550} & 168.43 & 89.43 & 46.93 & 25.14 & 11.86 & 6.50 & 2.79 & 0.68 & 1.30 & 1.84 & - \\
\hline & 11.04 & 9.45 & 7.02 & 6.25 & 4.02 & 3.39 & 1.92 & 0.73 & 1.83 & 2.46 & 0.648 \\
\hline
\end{tabular}

where $\tilde{\gamma}^{2}=(\bar{\mu}+b \rho \bar{\gamma} \gamma)^{2}+\bar{\gamma}^{2}$, and $\tilde{N}_{i}(\Delta t)$ is a Poisson random variable with parameter $\lambda \kappa \Delta t$.

The Continuous Time Limit Model is given in Proposition 2. 
Table 2

\section{Convergence of Percentage Errors in Option Prices}

The Table shows the percentage errors in option prices using the Euler and GARCH-Jump approximation schemes. The top entries show the convergence behavior of errors when the Euler approximation scheme is used. The bottom entries in each row show the errors when the approximating GARCH-Jump option model is used. The models are shown below where the relevant variables are defined in Proposition 4. The percentage error is defined as the GARCH (or Euler) price minus the true price divided by the true price, and reported as a percentage. The true price is the Euler price based on 1024 partitions per day. The parameter values used are discussed in the text. The option contracts are European calls with 30 days to expiration. In all cases 50000 simulations were used using antithetic control variables. The approximating GARCH-Jump Model defined in [Q1] for this case reduces to:

$$
\begin{aligned}
S_{i \Delta t}= & S_{(i-1) \Delta t} e^{\tilde{f}_{i \Delta t}(\Delta t)+\sqrt{h_{i \Delta t}} \tilde{J}_{i}(\Delta t) \sqrt{\Delta t}} \\
\phi_{(i+1) \Delta t}-\phi_{i \Delta t}= & \left(\beta_{0}+\beta_{2} q_{2}+\beta_{3} q_{3}\right) \Delta t+\phi_{i \Delta t}\left(\beta_{1}-1\right) \Delta t \\
& +\beta_{2}\left[\left|\frac{\tilde{J}_{i}(\Delta t)+(b \rho-\lambda \bar{\mu}) \sqrt{\Delta t}}{\sqrt{1+\lambda \hat{\gamma}^{2}}}\right|-q_{2}\right] \sqrt{\Delta t} \\
& +\beta_{3}\left[\max \left(-\frac{\tilde{J}_{i}(\Delta t)+(b \rho-\lambda \bar{\mu}) \sqrt{\Delta t}}{\sqrt{1+\lambda \hat{\gamma}^{2}}}, 0\right)-q_{3}\right] \sqrt{\Delta t} \\
h_{(i+1) \Delta t}= & \phi_{(i+1) \Delta t}^{2}
\end{aligned}
$$

\begin{tabular}{|c|c|c|c|c|c|c|c|c|c|c|c|}
\hline \multicolumn{12}{|c|}{ Number of Partitions per Day } \\
\hline Strike & 1 & 2 & 4 & 8 & 16 & 32 & 64 & 128 & 256 & 512 & 1024 \\
\hline \multirow{2}{*}{450} & 11.05 & 5.56 & 2.90 & 1.47 & 0.70 & 0.39 & 0.09 & 0.07 & 0.01 & 0.02 & - \\
\hline & -0.85 & -0.90 & -0.53 & -0.54 & -0.36 & -0.47 & -0.25 & -0.09 & -0.01 & 0.01 & -0.02 \\
\hline \multirow{2}{*}{460} & 14.19 & 7.24 & 3.81 & 1.96 & 0.95 & 0.53 & 0.12 & 0.09 & 0.01 & 0.04 & - \\
\hline & -1.26 & -1.30 & -0.79 & -0.76 & -0.52 & -0.68 & -0.35 & -0.14 & -0.03 & 0.01 & -0.02 \\
\hline \multirow{2}{*}{470} & 18.11 & 9.36 & 4.92 & 2.57 & 1.25 & 0.69 & 0.13 & 0.11 & 0.00 & 0.05 & - \\
\hline & -1.81 & -1.81 & -1.18 & -1.09 & -0.75 & -0.95 & -0.51 & -0.22 & -0.06 & 0.02 & -0.05 \\
\hline \multirow{2}{*}{480} & 23.02 & 12.03 & 6.32 & 3.35 & 1.64 & 0.91 & 0.15 & 0.17 & 0.00 & 0.06 & - \\
\hline & -2.51 & -2.45 & -1.68 & -1.46 & -1.00 & -1.27 & -0.72 & -0.30 & -0.07 & 0.05 & -0.09 \\
\hline \multirow{2}{*}{490} & 29.15 & 15.29 & 8.05 & 4.30 & 2.08 & 1.17 & 0.19 & 0.25 & 0.06 & 0.07 & - \\
\hline & -3.37 & -3.25 & -2.31 & -1.91 & -1.32 & -1.66 & -0.97 & -0.39 & -0.06 & 0.10 & -0.15 \\
\hline \multirow{2}{*}{500} & 36.71 & 19.26 & 10.16 & 5.46 & 2.58 & 1.45 & 0.22 & 0.33 & 0.10 & 0.10 & - \\
\hline & -4.38 & -4.23 & -3.03 & -2.49 & -1.73 & -2.14 & -1.30 & -0.52 & -0.07 & 0.15 & -0.22 \\
\hline \multirow{2}{*}{510} & 45.97 & 24.08 & 12.68 & 6.79 & 3.24 & 1.84 & 0.30 & 0.37 & 0.10 & 0.14 & - \\
\hline & -5.60 & -5.39 & -3.85 & -3.19 & -2.19 & -2.72 & -1.60 & -0.68 & -0.14 & 0.14 & -0.28 \\
\hline \multirow{2}{*}{520} & 57.32 & 29.86 & 15.71 & 8.31 & 4.02 & 2.27 & 0.34 & 0.37 & 0.03 & 0.18 & - \\
\hline & -7.04 & -6.78 & -4.76 & -4.04 & -2.80 & -3.36 & -1.93 & -0.90 & -0.26 & 0.07 & -0.32 \\
\hline \multirow{2}{*}{530} & 71.29 & 36.75 & 19.33 & 10.15 & 4.89 & 2.83 & 0.43 & 0.44 & 0.03 & 0.32 & - \\
\hline & -8.58 & -8.36 & -5.68 & -4.99 & -3.49 & -4.08 & -2.25 & -1.15 & -0.37 & -0.02 & -0.32 \\
\hline \multirow{2}{*}{540} & 88.28 & 44.86 & 23.51 & 12.17 & 5.77 & 3.36 & 0.54 & 0.47 & -0.01 & 0.36 & - \\
\hline & -10.34 & -10.20 & -6.75 & -6.14 & -4.31 & -4.94 & -2.64 & -1.40 & -0.52 & -0.21 & -0.44 \\
\hline \multirow{2}{*}{550} & 109.09 & 54.49 & 28.35 & 14.41 & 6.63 & 3.92 & 0.72 & 0.42 & 0.06 & 0.32 & - \\
\hline & -12.23 & -12.14 & -7.96 & -7.42 & -5.25 & -5.77 & -3.07 & -1.76 & -0.71 & -0.35 & -0.62 \\
\hline
\end{tabular}

where $\tilde{J}_{i}(\Delta t)=\tilde{X}_{i}^{(0)}+\Sigma_{j=1}^{\tilde{N}_{i}(\Delta t)} \tilde{X}_{i}^{(j)}(\Delta t), \tilde{X}_{i}^{(0)} \sim N(0,1), \tilde{X}_{i}^{(j)}(\Delta t) \sim N\left(\frac{\bar{\mu}+b \rho \gamma \bar{\gamma}}{\sqrt{\Delta t}}, \frac{\bar{\gamma}^{2}}{\Delta t}\right)$ and $\tilde{N}_{i}(\Delta t)$ is a Poisson random variable with parameter $\lambda \kappa \Delta t$ and

$$
\begin{aligned}
\tilde{f}_{i \Delta t}(\Delta t) & =\left(r-\frac{h_{i \Delta t}}{2}\right) \Delta t+\lambda \kappa\left(1-K_{i}\left(h_{i \Delta t}\right)\right) \Delta t \\
K_{i}\left(h_{i \Delta t}\right) & =e^{\sqrt{h_{i \Delta t}}(\bar{\mu}+b \rho \gamma \bar{\gamma})+\frac{1}{2} h_{i \Delta t} \bar{\gamma}^{2}}
\end{aligned}
$$

The Continuous Time Limit Model is given in Proposition 4. 


\section{Figure 1 \\ Comparing Convergence Rates of GARCH to Continuous Time Limits for Case 1}

The graphs compare the percentage errors in option prices produced by the GARCH-Jump model with the Euler approximation scheme. The number of time increments per day are indicated on the $\mathrm{x}$-axis . 50,000 sample paths were used to construct the prices, and antithetic control variates were used. The true prices were taken as the prices produced by an Euler scheme using 2000 partitions per day. The left panel shows the convergence behavior for 30 days call contracts and the right panel shows the convergence rate. The underlying price at date 0 is 500 , and the option strikes are indicated. The parameter values correspond to those of our estimated model. The parameters were estimated from time series data on the S\&P 500 as outlined in Section 3.
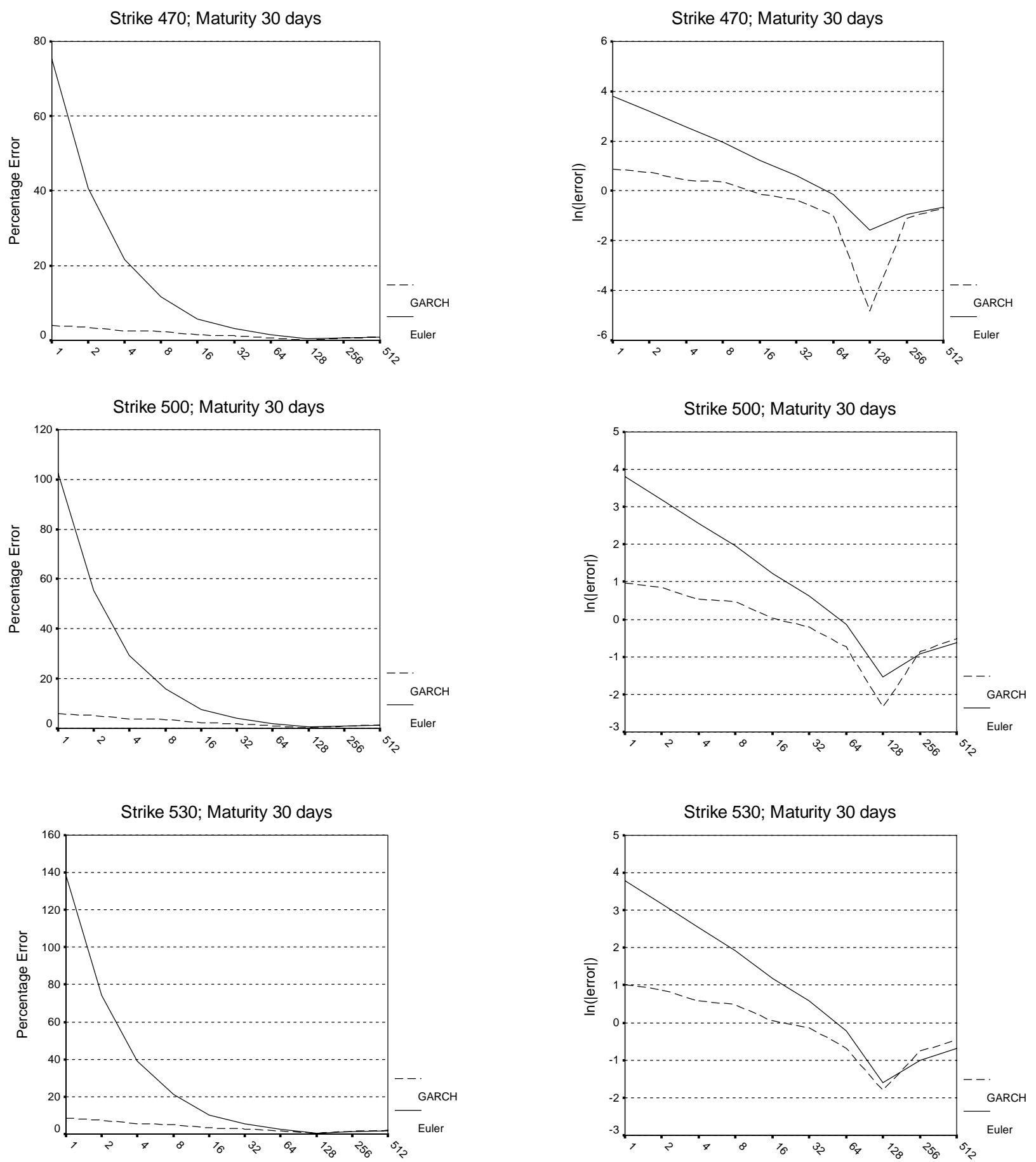


\section{Figure 2 \\ Comparing Convergence Rates of GARCH to Continuous Time Limits for Case 2}

The graphs compare the percentage errors in option prices produced by the GARCH-Jump model with the Euler approximation scheme. The number of time increments per day are indicated on the $\mathrm{x}$-axis . 50,000 simulations were used to construct the prices, and antithetic control variates were used. The true prices were taken as the prices produced by an Euler scheme using 2000 partitions per day. The left panel shows the convergence rate for 30 days call contracts and the right panel shows the convergence behavior. The underlying price at date 0 is 500 , and the option strikes are indicated. The parameter values correspond to those of our estimated model. The parameters were estimated from time series data on the S\&P 500 as outlined in Section 3.

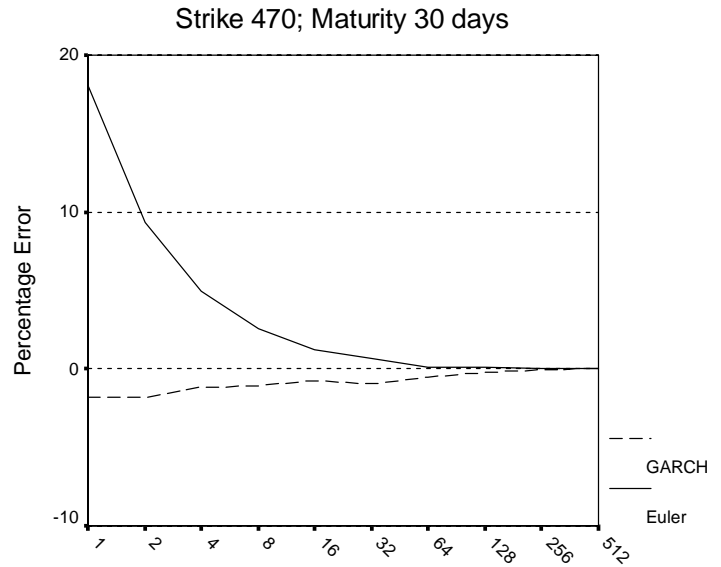

Strike 500; Maturity 30 days

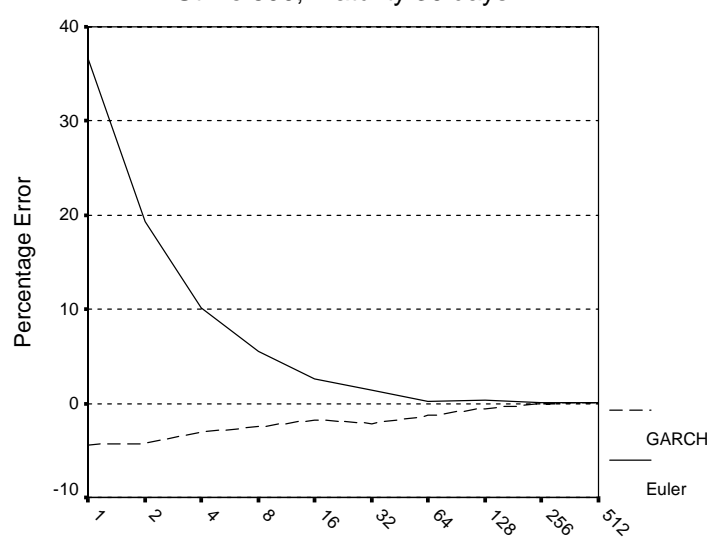

Strike 530; Maturity 30 days

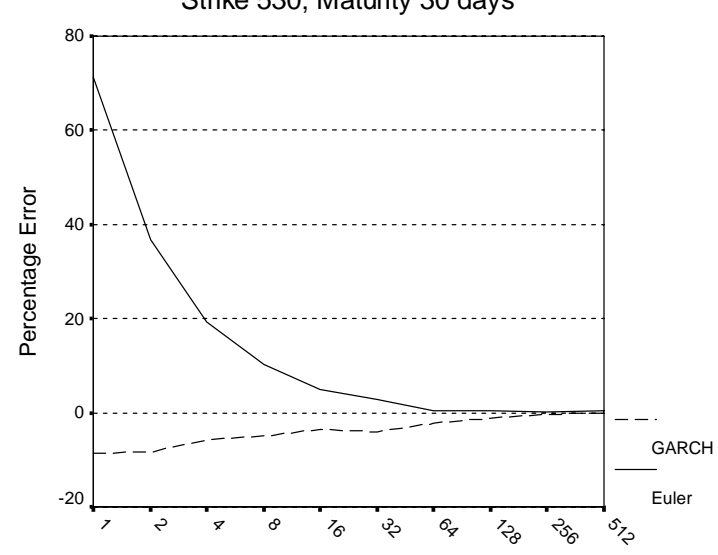

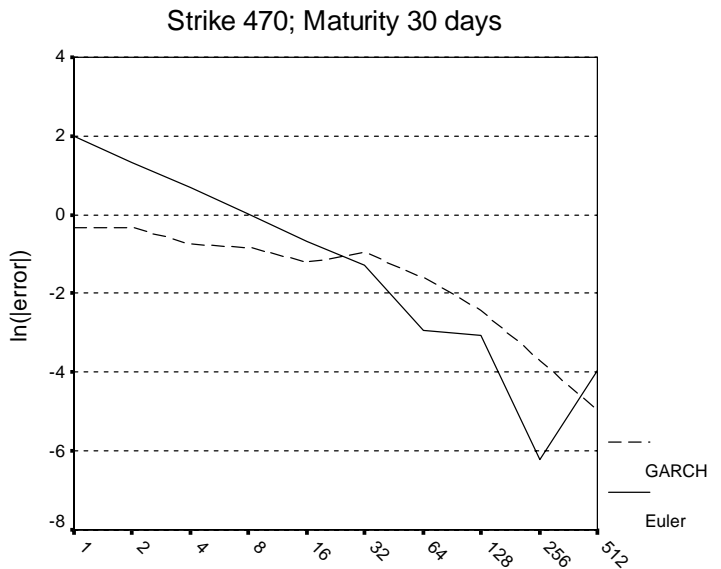

Strike 500; Maturity 30 days

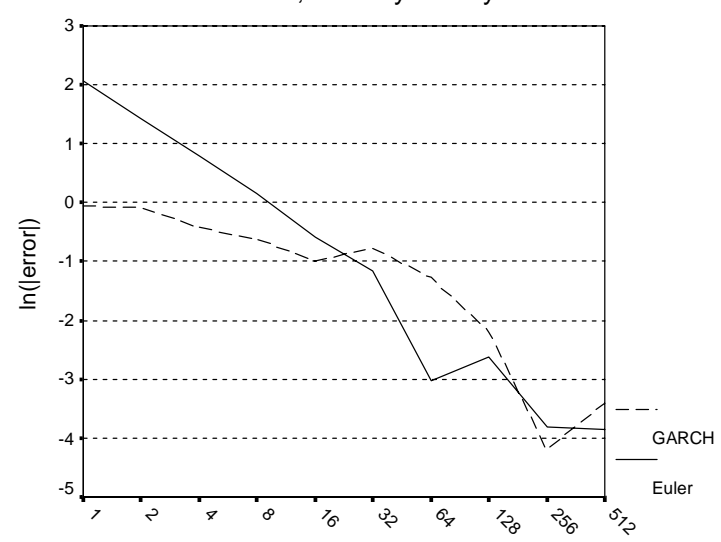

Strike 530; Maturity 30 days

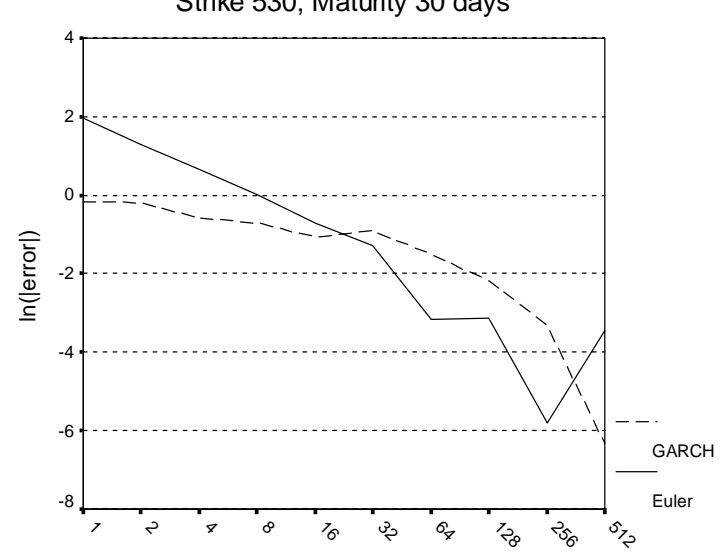

